This item was submitted to Loughborough's Research Repository by the author.

Items in Figshare are protected by copyright, with all rights reserved, unless otherwise indicated.

\title{
Calculation of radiative heat transfer in combustion systems
}

PLEASE CITE THE PUBLISHED VERSION

PUBLISHER

(C) Taylor \& Francis

VERSION

AM (Accepted Manuscript)

LICENCE

CC BY-NC-ND 4.0

REPOSITORY RECORD

Malalasekera, W., Hendrik K. Versteeg, Jonathan C. Henson, and J.C. Jones. 2019. "Calculation of Radiative Heat Transfer in Combustion Systems". figshare. https://hdl.handle.net/2134/5533. 
This item was submitted to Loughborough's Institutional Repository (https://dspace.lboro.ac.uk/) by the author and is made available under the following Creative Commons Licence conditions.

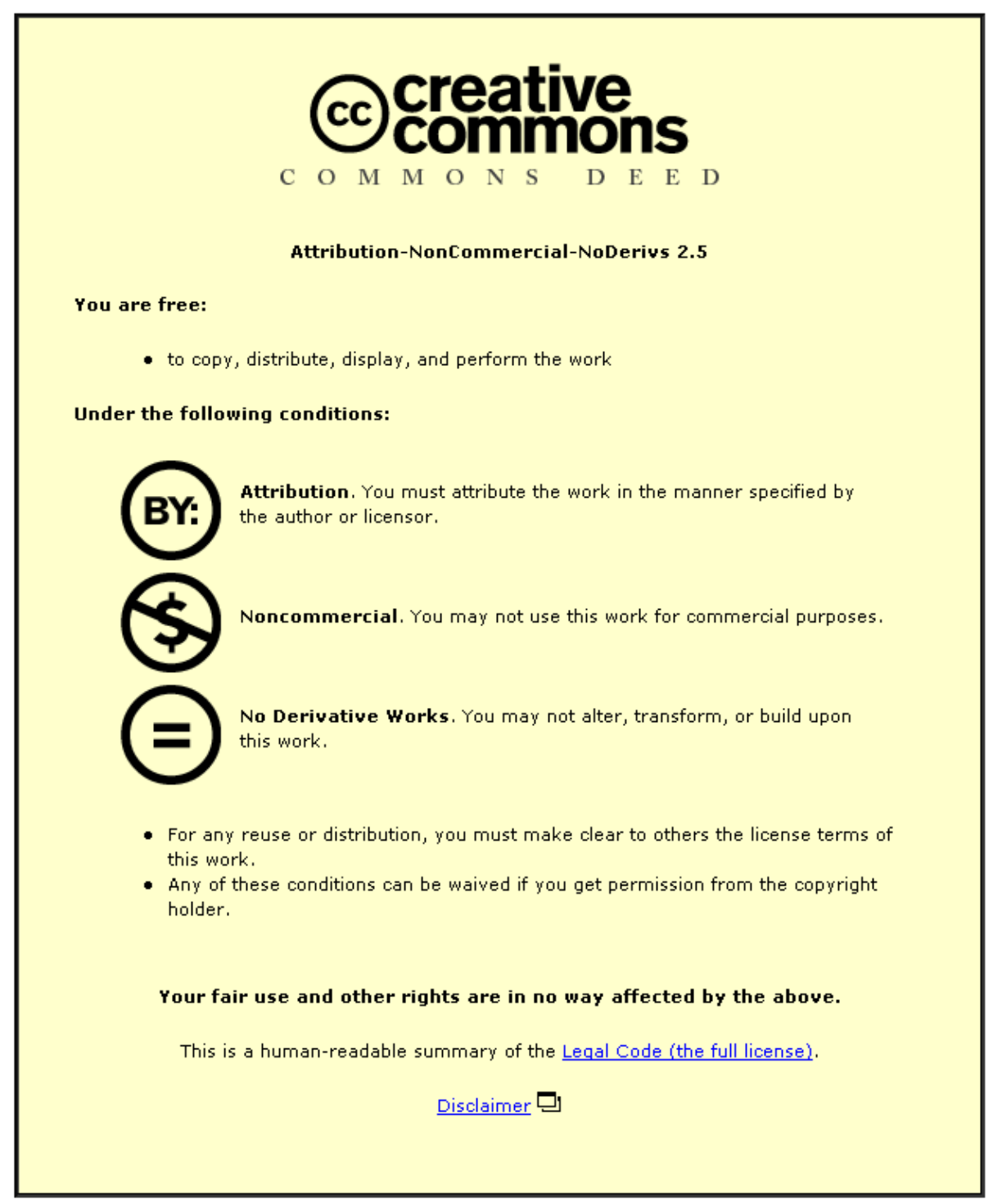

For the full text of this licence, please go to: http://creativecommons.org/licenses/by-nc-nd/2.5/ 


\title{
Calculation of Radiative Heat Transfer in Combustion Systems
}

W. MALALASEKERA ${ }^{*}$, H.K.VERSTEEG, J.C. HENSON and J.C. JONES

Department of Mechanical Engineering

Loughborough University, Loughborough

Leics LE11 3TU

United Kingdom

\begin{abstract}
Most practical combustion systems involve complex geometry configurations and CFD techniques used for the calculation of flow and combustion in such geometries use body-fitted non-orthogonal mesh systems. This paper reviews some of the currently available radiative heat transfer calculation techniques suitable for such CFD applications. The Monte Carlo method, the discrete transfer method, the YIX method, the discrete ordinates method and the finite volume method are discussed and some notable applications related to combustion problems are reviewed. Comparative results using all the methods outlined are presented for bench mark problems and their applicability to complex geometry situations are discussed. Radiative heat flux predictions for an S.I. engine simulation are presented to demonstrate the capability of the discrete transfer method in a pent-roof complex geometry combustion chamber. The paper also describes a ray based technique for the handling of turbulence-radiation interactions in combustion and its application is demonstrated in the prediction of a methane diffusion flame.
\end{abstract}

Key words: Radiation, Monte Carlo, discrete transfer, discrete ordinates, YIX, combustion.

\section{INTRODUCTION}

Over the last decade Computational Fluid Dynamics (CFD) and numerical combustion modelling techniques have advanced at a rapid rate making CFD and combustion modelling a useful tool for combustion equipment design. Many practical combustion systems, where radiation is the principal heat transfer mechanism, involve complex geometry. Initially complex geometry configurations were simplified by means of stepped approximations of the boundary shape. More recently, however, methodologies have been developed to handle geometric details. Body-fitted co-ordinate systems and their geometric flexibility have led the development of CFD techniques for complex geometries, Peric (1985), Rodi et al (1989), Shyy et al (1988). These have now been widely used in industry for the prediction of flow, combustion and heat transfer in practical engineering systems. Until recently, most body-fitted CFD techniques used hexahedral type control volume discretisations based on structured non-orthogonal grids. Even though there are well-established techniques for grid generation and well-known packages 
available, the requirement of a structured mesh make the grid generation task very time consuming for certain geometries. This has led to the development of block-structured and unstructured CFD techniques, Jiang and Przekwas (1994), Thomadakis and Leschziner (1996), Mathur and Murthy (1997). The latter utilise finite element type control volumes so that all geometrical details can be incorporated without having to pay much attention to mesh generation. These techniques have now advanced to the extent that they can handle non-regular meshes and mixed volume shapes. Most commercial CFD codes are now using unstructured methodology and the techniques are gaining increased popularity, because of the exceptional geometric flexibility.

Although rapid advances have been made in CFD research to include unstructured techniques, other modelling areas such as radiation have been slow to adapt to these emerging developments. The authors have been developing and evaluating radiative heat transfer techniques particularly for the use in complex geometry combustion applications. This paper reviews some of the currently available radiative transfer techniques for such applications and our predictive experience with these techniques. Particular attention is given to methods, which can be used in conjunction with body-fitted and unstructured grids and are, therefore, suitable for application within combustion prediction procedures and problems involving complex geometry. We only review the more recent developments.

In the combustion field various models that include advanced chemistry and turbulence effects are being used at the present time. To realise the potential and the accuracy promised by these calculation techniques the computation of heat transfer, and in particular radiation, requires a great deal of attention, otherwise it is not possible to expect accurate results for emissions and other flow properties. One of the important issues concerning combustion modelling is the inclusion of turbulence/radiation interactions. There are various models to handle the effects of turbulence/chemistry interactions in combustion but less work has been done to incorporate turbulence/radiation interactions in combustion. Most coupled radiation calculations involving combustion use averaged temperature, which is not satisfactory if accurate prediction of species concentrations are required. In this paper, we briefly discuss a technique, which we have been developing to address the issue of incorporating turbulence/radiation interactions.

Another important topic concerning radiative heat transfer modelling is the calculation of radiative properties. Until recently, the use of the weighted sum of grey gases approximation of Hottel and various empirical correlations has been the standard practice for calculating radiative 
properties in many applications. But recent evaluation studies have shown that accurate calculation of radiative properties is very important and the radiative heat transfer results depends very much on the radiative property values. There are several advanced techniques available to the user today for calculating radiative properties of a combustion media. A full review of these techniques is beyond the scope of this paper. The interested user is referred to Hsieh and Greif (1972), Edwards and Balakrisnan (1973), Denison and Webb (1993, 1995), Li et al (1995), Bressloff et al (1996a), Lallemant and Webber (1996), Carvalho and Farias (1998) and Henson (1998) for further details of radiative property calculation algorithms.

In the following sections of the paper we outline the methods, which we consider suitable for modern-day CFD applications. Essential equations to describe each method are presented and advantages and disadvantages of each technique are discussed based on our predictive experience and some applications related to combustion problems are given. Comparative results using all the methods applicable to complex geometry combustor configurations are presented and the level of accuracy and the factors affecting accuracy are briefly discussed. Radiative heat flux predictions for an S.I. engine combustion chamber are presented to demonstrate the use of discrete transfer method in a complex, time-dependent geometry. We also present results of our turbulence-radiation interaction modelling work and illustrate its applicability to combustion related problems.

\section{GOVERNING EQUATIONS OF RADIATIVE TRANSFER}

The general relationship that governs the radiative heat transfer in the presence of emitting/absorbing and scattering medium in its most general form is given by

$$
\frac{d I(\mathbf{r}, \hat{\mathbf{s}})}{d s}=\hat{\mathbf{s}} . \nabla I(\mathbf{r}, \hat{\mathbf{s}})=\kappa I_{b}(\mathbf{r})-\beta I(\mathbf{r}, \hat{\mathbf{s}})+\frac{\sigma_{s}}{4 \pi} \int_{4 \pi} I\left(\hat{\mathbf{s}}_{i}\right) \Phi\left(\hat{\mathbf{s}}_{i}, \hat{\mathbf{s}}\right) d \Omega_{i}
$$

where $I(\mathbf{r}, \hat{\mathbf{s}})$ is the radiation intensity at a given location $\mathbf{r}$, in the direction $\hat{\mathbf{s}}$ within a small pencil of rays, travelling through a participating medium (see Modest, 1993). The radiative transfer equation (RTE) can be rewritten in terms of non-dimensional optical co-ordinates:

$$
\tau=\int_{0}^{s}\left(\kappa+\sigma_{s}\right) d s=\int_{0}^{s} \beta d s
$$

and a single scattering albedo defined as

$$
\omega=\frac{\sigma_{s}}{\kappa+\sigma_{s}}=\frac{\sigma_{s}}{\beta}
$$


The transfer equation can be re-written as

$$
\frac{d I(\mathbf{r}, \hat{\mathbf{s}})}{d \tau}=-I(\mathbf{r}, \hat{\mathbf{s}})+(1-\omega) I_{b}(\mathbf{r})+\frac{\omega}{4 \pi} \int_{4 \pi} I\left(\hat{\mathbf{s}}_{i}\right) \Phi\left(\hat{\mathbf{s}}_{i}, \hat{\mathbf{s}}\right) d \Omega_{i}
$$

By defining the source function as

$$
S(\mathbf{r}, \hat{\mathbf{s}})=(1-\omega) I_{b}(\mathbf{r})+\frac{\omega}{4 \pi} \int_{4 \pi} I\left(\hat{\mathbf{s}}_{i}\right) \Phi\left(\hat{\mathbf{s}}_{i}, \hat{\mathbf{s}}\right) d \Omega_{i}
$$

the transfer equation becomes

$$
\frac{d I(\mathbf{r}, \hat{\mathbf{s}})}{d \tau}+I(\mathbf{r}, \hat{\mathbf{s}})=S(\mathbf{r}, \hat{\mathbf{s}})
$$

\section{Boundary Conditions}

For difussively emitting and reflecting opaque surfaces the outgoing intensity may be written as

$$
I\left(\mathbf{r}_{w}, \hat{\mathbf{s}}\right)=I\left(\mathbf{r}_{w}\right)=J\left(\mathbf{r}_{w}\right) / \pi=\varepsilon_{w} I_{b}\left(\mathbf{r}_{w}\right)+\rho_{w} H\left(\mathbf{r}_{w}\right) / \pi
$$

where $J\left(\mathbf{r}_{w}\right)$ is the radiosity and $H\left(\mathbf{r}_{w}\right)$ is the hemispherical irradiation from all incoming directions $\hat{\mathbf{s}}^{\prime}$ at the wall.

$$
I\left(\mathbf{r}_{w}, \hat{\mathbf{s}}\right)=\varepsilon_{w} I_{b}\left(\mathbf{r}_{w}\right)+\frac{\rho_{w}}{\pi} \int_{\hat{\mathbf{n}} . \hat{s}^{\prime}<0} I\left(\mathbf{r}_{w}, \hat{\mathbf{s}}^{\prime}\right)\left|\hat{\mathbf{n}} . \hat{\mathbf{s}}^{\prime}\right| d \Omega^{\prime}
$$

This is the most widely used boundary condition in combustion related radiative transfer problems. Expressions for other boundary conditions can be found in Modest (1993).

\section{SOLUTION METHODS}

Exact analytical solutions to the radiative transfer equation (RTE) are not available except in a small number of idealised cases. In most practical problems further simplification of the transfer equation is not possible. In particular, the boundary conditions are dictated by the problem geometry and full three-dimensional effects and all angular directions must be considered in solving the transfer equation. Further complications arise in combusting systems, because the equation of transfer contains terms involving the unknown temperature field and radiative properties, which are dependent on temperature and concentration. Combustion effects dominate the temperature field and the radiative properties of the medium should be determined from the concentration of species of combustion. Due to this intricate coupling of temperature and radiative properties of the medium the solution of the RTE in combustion problems becomes very complex. To add to this complexity the properties of combustion products are non-grey and 
therefore, unless spectrally resolved radiation calculations are performed, property calculation models are required to model the spectral effects.

Over the past years a number of methods have been developed for the solution of radiative heat transfer. These include various analytical approximation techniques and a suite of numerical methods. Some early methods have now been abandoned because of their limited applicability to general situations involving radiative transfer. In this paper we review and discuss some of the currently used methods and their applicability to combustion systems with complex geometry. Other methods, such as the zone method, P-N methods, flux methods and finite element methods are not discussed here. Details can be found in the reviews and texts by Sarofim (1986), Viskanta and Mengüç (1987), Chan (1987), Howell (1988), Siegel and Howell (1992), Modest (1993), Carvalho and Farias (1998) and Maruyama and Guo (2000). We concentrate on the Monte Carlo method (Howell and Perlmutter, 1964), the discrete transfer method (Lockwood and Shah, 1981), discrete ordinate methods (Chandrasekhar, 1960, Hyde and Truelove, 1977, Fiveland, 1982), the YIX method (Tan and Howell, 1990 and Hsu et al, 1992, 1993) and the Finite Volume method (Chui and Raithby, 1993). Due to historical reasons various research groups within the combustion community have usually adopted one method and stayed with the selected technique for coupling radiation to the combustion calculation. The advancement of the computer hardware and availability of commercial software, incorporating several alternative techniques, now offers the user a selection of radiative heat transfer techniques. In this paper we attempt to review the most widely available techniques. Within our group we have implemented all the techniques except the finite volume method that are discussed in this paper and summarise our predictive experience. The results that are presented for comparison have been obtained with codes running on the same computing platform, and, where relevant, using the same ray tracing method. This enables a realistic comparison of the computational efficiencies of various techniques. Much of the mathematical detail of each technique is not presented in detail in the interest of brevity; the reader is pointed to cited references for such details.

\section{The Monte Carlo Method}

In the Monte Carlo (MC) ray tracing method the radiative heat transfer is calculated by randomly releasing a statistically large number of energy bundles and tracking their progress through the participating medium. The medium is usually discretised into volume and surface elements for calculation purposes. The method is independent of the co-ordinates system and therefore applicable to arbitrary shaped and complex configurations. Several algorithms have been proposed (see Farmer, 1995 and the recent review by Howell, 1998). We have used the path- 
length based scheme of Farmer (1995) which is a computationally efficient version with essentially three distinguishing features: (i) the power content of a bundle is partitioned into the sub-volumes traversed by the bundle along its path such that the absorbed fraction in any volume is:

Absorbed fraction $=1-e^{-\kappa \delta s}$

where $\delta \mathrm{s}$ is the path length in each subvolume with homogeneous absorption coefficient, $\kappa$. (ii) The probable distance a bundle travels before a scattering collision is determined from a cumulative distribution function. A random number $R$ is selected from a uniform distribution between 0 and 1 to determine the scattering path length as:

$$
s=-\ln R / \sigma_{s}
$$

In non-homogeneous media the scattering coefficient, $\sigma_{s}$ varies along the bundle path, although usually its value is presumed constant over each subvolume. A summation is then taken for each subvolume $(n)$ through which the bundle travels such that a scattering collision only occurs when:

$$
\sum_{n} \sigma_{s, n} \delta s_{n}>-\ln R
$$

(iii) The absorption calculation in Eq. (9) is only performed as a bundle exits a subvolume. When multiple scattering events occur within a single subvolume, the path length is accumulated, rather than evaluating the expensive exponential term after each collision, resulting in a significant speed-up. Other aspects of this path-length based algorithm, such as modelling the bundles' emission and scattering behaviour, are identical to the traditional collision-based Monte Carlo method and can be found in Modest (1993). For the application of the Monte Carlo technique to nonorthogonal geometries, special treatment is required to select the bundle emission points within the irregular subsurfaces and subvolumes that arise from the body-fitted co-ordinate system. A finite element parametric mapping technique is used to randomly determine each starting location by mapping a uniform distribution onto the irregular sub region. First, random numbers $\left(R_{x}, R_{y}, R_{z}\right)$ are selected for each coordinate direction and then these are used to derive appropriate bilinear shape functions, $\psi_{n}$. An emission point, $x$ is then interpolated from the appropriate surface or volume nodal co-ordinates, $x_{n}\left(x_{n}, y_{n}, z_{n}\right)$ from the expression:

$$
x=\sum \psi_{n} x_{n}
$$




\section{The Discrete Transfer Method}

In the Discrete Transfer Method (DTM), Lockwood and Shah, (1981), the solution proceeds by first discretising the radiation space into homogeneous surface and volume elements. Rays are emitted from the centre of each boundary subsurface, with position vector $\mathbf{r}$, in directions determined by discretising the $2 \pi$ hemispherical solid angle above the surface into $n$ finite solid angles, $\delta \Omega$. Shah (1979) chose to divide the hemisphere into $N_{\theta}$ equal polar angles and $N_{\phi}$ equal azimuthal angles such that $N_{\Omega}=N_{\theta} \cdot N_{\phi}$ and:

$$
\delta \theta=\frac{\pi}{2 N_{\theta}}, \quad \delta \phi=\frac{2 \pi}{N_{\phi}}
$$

A ray is traced through the centre of each solid angle (subdivision), in the direction $-\hat{\mathbf{s}}_{k}$, until it strikes a boundary at $\mathbf{r}_{L}=\mathbf{r}-\hat{\mathbf{s}}_{k} L_{k}$, such that the ray path length is $L_{k}=\left|\mathbf{r}-\mathbf{r}_{L}\right|$. Then, starting at $\mathbf{r}_{L}$, the ray is followed back to its origin $\mathbf{r}$, while solving for the intensity distribution along its path with the recurrence relation:

$$
I_{n+1}=I_{n} e^{-\delta s^{*}}+S\left(1-e^{-\delta s^{*}}\right)
$$

where $n$ and $n+1$ designate successive boundary locations, separated by a distance $\delta s=\delta \mathrm{s}^{*} / \beta$, as the ray passes through each control volume. The source function, $S$ and the extinction coefficient, $\beta$ are both assumed constant over the interval. Eq. (14) is applied in the direction towards the origin of each ray, and the radiative heat flux contributions are summed over all solid angles, assuming that the intensity is constant over each finite solid angle. This gives the incident flux for the subsurface:

$$
q_{-}=\sum_{N_{\Omega}} I_{i}(\hat{\mathbf{s}}) \hat{\mathbf{s}} \cdot \hat{\mathbf{n}} \delta \Omega
$$

An initial intensity value $I_{o}=q_{+} / \pi$ is used for each subsurface, where the emitted flux, $q_{+}$is given by:

$$
q_{+}=(1-\varepsilon) q_{-}+\varepsilon E_{s}
$$

Since $q_{+}$depends on the value of $q_{-}$, an iterative solution is required, unless the surfaces are black $(\varepsilon=1)$.The net radiative heat flow for each subsurface, area $A_{i}$, is then:

$$
Q_{s i}=A_{i}\left(q_{+}-q_{-}\right)
$$

Solution of Eq. (14) also requires a value for the source function $S$ where: 


$$
S=(1-\omega) I_{b}+\frac{\omega}{4 \pi} \int_{4 \pi} I\left(\hat{\mathbf{s}}_{i}\right) \Phi\left(\hat{\mathbf{s}}, \hat{\mathbf{s}}_{i}\right) d \Omega_{i}
$$

A cell averaged (directionally independent) value is taken for $S$. Though this considerably simplifies the analysis, scattering anisotropy cannot be described. DTM computes the radiative sources via an energy balance over the medium elements. Lockwood and Shah (1981) consider each ray as a beam of radiative energy, such that the radiative source associated with its passage through a volume $n$ (from the definition of intensity) is:

$$
\begin{aligned}
\delta Q_{g}\left(\hat{\mathbf{s}}_{k}\right) & =\int_{\delta \Omega}\left(I_{n+1}-I_{n}\right) \delta A_{s}\left(-\hat{\mathbf{s}}_{k} \cdot \hat{\mathbf{n}}\right) d \Omega_{k} \\
& =\left(I_{n+1}-I_{n}\right) \delta A_{s} \cos \theta_{k} \sin \theta_{k} \sin (\delta \theta) \delta \phi_{k}
\end{aligned}
$$

where $\delta A_{s}$ is the area of the surface element from which the ray was emitted. Summing the individual source contributions from all the $N$ rays passing through a volume element, and then dividing this value by its volume, $V$, gives the divergence of radiative heat flux as:

$$
\nabla \cdot \mathbf{q}_{r}(\mathbf{r})=\frac{1}{V} \sum_{i=1}^{N} \delta Q_{g, i}
$$

where $\mathbf{r}$ is anywhere inside the volume element $n$ over which the source is taken as constant.

\section{YIX Method}

The YIX method solves the integral equations of radiative heat transfer (Tan 1989). Written in angular-distance form for a three-dimensional, grey, emitting, absorbing and linear anisotropically scattering media they have the form of Eqs. (21-29) for the medium irradiation, $f_{g}$, the medium heat flux vector, $\boldsymbol{f}_{r}$, and the incident surface heat flux, $f_{s}$.

$$
\begin{array}{ccc}
f_{g}(\mathbf{r})=\frac{1}{\pi} \int_{0}^{4 \pi}\left\{\int_{0}^{L(\mathbf{r}, \hat{\mathbf{s}})} \exp \left[-\int_{0}^{t} \beta\left(\mathbf{r}-\hat{\mathbf{s}} t^{\prime}\right) d t^{\prime}\right]\left[F_{g}\left(\mathbf{r}_{t}\right)+\boldsymbol{F}_{r}\left(\mathbf{r}_{t}\right) \cdot \hat{\mathbf{s}}\right] d t+\right. & \mathbf{r} \in V \\
\left.+\exp \left[-\int_{0}^{L(\mathbf{r}, \hat{\mathbf{s}})} \beta(\mathbf{r}-\hat{\mathbf{s}} t) d t\right] F_{s}\left(\mathbf{r}_{L}\right)\right\} d \Omega(\mathbf{r}) & \\
\boldsymbol{f}_{r}(\mathbf{r})=\frac{1}{\pi} \int_{0}^{4 \pi}\left\{\int_{0}^{L(\mathbf{r}, \hat{\mathbf{s}})} \exp \left[-\int_{0}^{t} \beta\left(\mathbf{r}-\hat{\mathbf{s}} t^{\prime}\right) d t^{\prime}\right]\left[F_{g}\left(\mathbf{r}_{t}\right)+\boldsymbol{F}_{r}\left(\mathbf{r}_{t}\right) \cdot \hat{\mathbf{s}}\right] d t+\right. & \mathbf{r} \in V \\
\left.+\exp \left[-\int_{0}^{L(\mathbf{r}, \hat{\mathbf{s}})} \beta(\mathbf{r}-\hat{\mathbf{s}} t) d t\right] F_{s}\left(\mathbf{r}_{L}\right)\right\} \hat{\mathbf{s}}^{T} d \Omega(\mathbf{r}) &
\end{array}
$$




$$
\begin{aligned}
f_{s}(\mathbf{r})=\frac{1}{\pi} \int_{0}^{2 \pi}\left\{\int_{0}^{L(\mathbf{r}, \hat{\mathbf{s}})} \exp \left[-\int_{0}^{t} \beta\left(\mathbf{r}-\hat{\mathbf{s}} t^{\prime}\right) d t^{\prime}\right]\left[F_{g}\left(\mathbf{r}_{t}\right)+\boldsymbol{F}_{r}\left(\mathbf{r}_{t}\right) \cdot \hat{\mathbf{s}}\right] d t+\right. & \mathbf{r} \in S \\
\left.+\exp \left[-\int_{0}^{L(\mathbf{r}, \hat{\mathbf{s}})} \beta(\mathbf{r}-\hat{\mathbf{s}} t) d t\right] F_{s}\left(\mathbf{r}_{L}\right)\right\}(-\hat{\mathbf{s}} \cdot \hat{\mathbf{n}}) d \Omega(\mathbf{r}) &
\end{aligned}
$$

where the terms:

$$
\begin{aligned}
& f_{g}(\mathbf{r})=4 e_{g}(\mathbf{r})-\frac{1}{(1-\omega) \beta} \nabla \cdot \mathbf{q}_{r}(\mathbf{r}) \\
& F_{g}(\mathbf{r})=e_{g}(\mathbf{r})-\frac{\omega}{4(1-\omega) \beta} \nabla \cdot \mathbf{q}_{r}(\mathbf{r}) \\
& \boldsymbol{f}_{r}(\mathbf{r})=\mathbf{q}_{r}(\mathbf{r})=\left(\mathbf{q}_{x}, \mathbf{q}_{y}, \mathbf{q}_{z}\right)^{T} \\
& \boldsymbol{F}_{r}(\mathbf{r})=\frac{\omega g}{4} \mathbf{q}_{r}(\mathbf{r}) \\
& f_{s}(\mathbf{r})=e_{s}(\mathbf{r})-\frac{1}{\varepsilon} q_{s}(\mathbf{r}) \\
& F_{s}(\mathbf{r})=e_{s}(\mathbf{r})-\frac{1-\varepsilon}{\varepsilon} q_{s}(\mathbf{r})
\end{aligned}
$$

Here, $e_{g}, \beta$ and $\omega$ are, respectively, the medium blackbody emissive power, medium extinction and scattering albedo; $e_{g}, \varepsilon$ and $q_{s}$ are the surface blackbody emissive power, surface emissivity and net radiative surface heat flux, and $g$ is a coefficient of the linear anisotropic scattering phase function.

A YIX solution proceeds by discretising the radiation space into homogeneous surface and volume elements and solving for the radiative transfer at points located at their geometric centres. These are either within the medium, where $\mathbf{r} \in V$, or on the enclosing surface, where $\mathbf{r} \in S$. The solid angle about $\mathbf{r}$ is discretised into finite solid angles. Normal practice is to use an $S_{N}$ discrete ordinate quadrature (Lathrop and Carlson, 1965). A ray is then traced through the centroid of each solid angle element, in the direction $-\hat{\mathbf{s}}$, until it strikes a boundary at $\mathbf{r}_{L}=\mathbf{r}-\hat{\mathbf{s}} L$, such that the ray path length is $L(\mathbf{r}, \hat{\mathbf{s}})=\left|\mathbf{r}-\mathbf{r}_{L}\right|$. The distance integral at $\mathbf{r}$ associated with the ordinate $\hat{\mathbf{s}}$ is then computed using a nonuniform equal-weights quadrature (in optical thickness). This is expressed as: 


$$
\begin{aligned}
\int_{0}^{L(\mathbf{r}, \hat{\mathbf{s}})} \exp \left[-\int_{0}^{t} \beta\left(\mathbf{r}-\hat{\mathbf{s}} t^{\prime}\right) d t^{\prime}\right] F\left(\mathbf{r}_{t}\right) d t & \cong \lambda\left[F\left(t_{0}\right)+\sum_{i=1}^{n-1} F\left(t_{i}\right)\right]+ \\
+ & {\left[P_{n+1}+\Gamma_{L} \cdot Q_{n+1}\right] F\left(t_{n}\right) }
\end{aligned}
$$

where the appropriate function $F(t)$ is evaluated at points $\mathbf{r}_{i}=\mathbf{r}-\hat{\mathbf{s}} t_{i}(i=0,1, \ldots, n)$ along the ray-path, with total optical length $\Gamma_{L} \cdot \lambda, t_{i}, Q_{i}, P_{i}$ are quadrature constants computed once at the start of the calculation and stored. The user specifies the number of integration points $\mathbf{r}_{i}$, and therefore the accuracy of the exponential integration of Eq. (30), via the value $\lambda$ or a related parameter. Hence, in summary, the terms $f_{g}, \boldsymbol{f}_{r}$, and $f_{s}$ are constructed by first assembling the distance integrals for each ordinate direction $\hat{\mathbf{s}}$, and subsequently, combining them in a weighted summation over the $4 \pi$ solid angle at medium points and $2 \pi$ solid angle at surface points. The reader is referred to Tan (1989) and Hsu et al. (1993) for further details of the scheme and its application.

\section{Discrete Ordinates Method}

In the discrete ordinates method the equation of transfer is solved for a set of $n$ different directions in the total of $4 \pi$ solid angle and the integrals over directions are replaced by numerical quadrature. Thus the equation of transfer is approximated by

$$
\hat{\mathbf{s}}_{i} . \nabla I\left(\mathbf{r}, \hat{\mathbf{s}}_{i}\right)=\kappa I_{b}(\mathbf{r})-\beta I\left(\mathbf{r}, \hat{\mathbf{s}}_{i}\right)+\frac{\sigma_{s}}{4 \pi} \sum_{j=1}^{n} w_{j} I\left(\mathbf{r}, \hat{\mathbf{s}}_{j}\right) \Phi\left(\mathbf{r}, \hat{\mathbf{s}}_{\mathbf{i}}, \hat{\mathbf{s}}_{\mathbf{j}}\right)
$$

where $w_{j}$ are quadrature weights associated with the directions $\hat{\mathbf{s}}_{i}$. The equation is subjected to the boundary condition

$$
I\left(\mathbf{r}_{w}, \hat{\mathbf{s}}_{i}\right)=\varepsilon_{w} I_{b}\left(\mathbf{r}_{w}\right)+\frac{\rho_{w}}{\pi} \sum_{\hat{\mathbf{n}} . \hat{s}_{j}<0} w_{j} I\left(\mathbf{r}_{w}, \hat{\mathbf{s}}_{j}\right)\left|\hat{\mathbf{n}}_{.} \hat{\mathbf{s}}_{j}\right| \quad \hat{\mathbf{n}} . \hat{\mathbf{s}}_{i}>0
$$

The ordinates $\hat{\mathbf{s}}_{i}$ and angular weights are available in the literature, Lathrop and Carlson (1965), Fiveland (1991) and the basis of obtaining the weights is discussed further in Modest (1993). The order of the $S_{N}$ approximation is denoted as $S_{2}, S_{4}, S_{6} \ldots . . S_{N}$. The total number of directions used ( $n$ ) is related to $N$ through the relation $n=N(N+2)$. The angular approximation transforms the original integro-differential equation into a set of coupled differential equations. The set of coupled differential equations are solved by discretisation using finite volume methods, see Modest (1993). 


$$
\xi_{i} \frac{d I_{i}}{d x}+\eta_{i} \frac{d I_{i}}{d y}+\mu_{i} \frac{d I_{i}}{d z}+\beta I_{i}=\beta S_{i} \quad \quad i=1,2, \ldots \ldots . n
$$

where $S_{i}$ is the source function and $\xi_{i}, \eta_{i}$ and $\mu_{i}$ are the direction cosines of direction $i$. The source function is defined as

$$
S_{i}=(1-\omega) I_{b}+\frac{\omega}{4 \pi} \sum_{j=1}^{n} w_{j} \Phi_{i j} I_{j} \quad i=1,2, \ldots \ldots . . n
$$

Equation (33) is discretised by integration over a control volume to obtain a relationship for volume averaged intensity $I_{P i}$

$$
I_{P i}=\frac{\beta V \gamma S_{P i}+\left|\xi_{i}\right| A_{x} I_{x_{i}, i}+\left|\eta_{i}\right| A_{y} I_{y_{i}, i}+\left|\mu_{i}\right| A_{z} I_{z_{i}, i}}{\beta V \gamma+\left|\xi_{i}\right| A_{x}+\left|\eta_{i}\right| A_{y}+\left|\mu_{i}\right| A_{z}}
$$

where $V$ is the volume of the cell, and $A_{x}, A_{y}$, and $A_{z}$ denote the areas of the cell faces normal to directions $x, y$ and $z$ respectively. The parameter $\gamma$ is a weighting factor that relates the incoming and outgoing boundary radiation intensities to the volume average intensities through

$$
\begin{aligned}
& I_{P i}=\gamma I_{x_{e, i}}+(1-\gamma) I_{x_{i, i}} \\
& I_{P i}=\gamma I_{y_{e, i}}+(1-\gamma) I_{y_{i, i}} \\
& I_{P i}=\gamma I_{z_{e, i}}+(1-\gamma) I_{z_{i, i}}
\end{aligned}
$$

subscript $i$ and $e$ denotes entering and exit faces. The weighting factor $\gamma$ is a constant $0 \leq \gamma \leq 1$ and this scheme is known as the weighted difference scheme. The most widely used Diamond and Step difference schemes are obtained by setting $\gamma$ to 0.5 and 1.0, respectively. Further details of the method and its derivation can be found in Modest (1993).

\section{The Finite Volume Method}

Raithby and co-workers (Raithby and Chui, 1990, Chui et al, 1992, 1993, Chui and Raithby, 1993) have presented a control volume integration method for the calculation of radiative heat transfer. Further modifications to this method have been proposed by several other researchers to demonstrate the application of the discrete ordinate like discretisation practices to complex geometry problems. The formulation of the method is very similar to the discrete ordinates method. Equations for intensity are solved for a set of discrete directions which span the total solid angle $4 \pi$. In addition to control volume integration the finite volume method uses a control angle integration as well (see Chai et al, 1994a,c). Further improvements to the original method along with appropriate interpolation techniques for non-orthogonal mesh systems are available for radiative heat transfer calculations in complex geometries. The difficulty, which arises in the 
application of this class of methods to non-orthogonal cells, is the evaluation of cell intensities and handling of control angle overhanging. This is caused by misalignment of cell surfaces with discretised control angles. Several techniques have been developed to overcome this problem; details can be found in Chai and Modar (1996), Baek et al (1998) and Murthy and Mathur (1998).

\section{SOME COMBUSTION RELATED APPLICATIONS}

The earliest general-purpose radiation models emerged at the time when CFD codes started to be applied widely to industrial problems where radiation is the main heat transfer mechanism, such as industrial furnaces and power station boilers. It is beyond the scope of this paper to give a comprehensive review of all the applications. We merely point out some of the most important examples. Early applications of integrated CFD/radiation models developed by the group at Imperial College, London include the studies by Abbas et al (1984) and Lockwood et al (1986a,b). Abbas et al predicted the flow, combustion and heat transfer in a refinery process heater. The radiative heat transfer was computed with the discrete transfer method. The work included a zoned treatment of the CFD mesh for the burner region and the combustor volume and also illustrated the use of special radiation meshes. Whilst the flow problem was solved on a $13 \times 25 \times 22$ mesh for the burner region and a $39 \times 25 \times 16$ for the combustor zone, the radiation problem was solved on a much coarser $8 \times 4 \times 16$ mesh. The broad features of flow and heat transfer were in 'remarkably good agreement' with measurements. Lockwood et al (1986a) have compared the results of calculations for the combustor aerodynamics against data, for the heat transfer and temperature distributions in a gas-fired combustor and for the particle trajectories in a coal-fired corner-fired power station combustion chamber. Radiation calculations used the discrete transfer method. Further application of this method in coal furnaces and combustors have been reported in Lockwood et al (1986b). Truelove (1984) demonstrated the use of the discrete ordinates method in modelling pulverised-coal burners and furnaces. No radiative heat flux results were reported but comparison of predicted and measured axial temperature data showed good agreement. The three-dimensional furnace modelling study of Boyd and Kent (1986) used the discrete transfer method with a coal combustion model to study a large scale 500 MWe power station combustor. The predicted temperature, oxygen volume fraction and radiative wall heat fluxes were compared with measurements obtained from the operating furnace. Given the complexity and scale of the problem the agreement between predictions and measurements was generally good. 
Carvalho and Coelho (1990) and Coelho and Carvalho (1995b) have reported a comprehensive programme of validation studies to explore the potential of CFD/radiation/combustion chemistry codes for the design of power station boilers. Both studied present three-dimensional models of Portuguese power station boilers and compare results with experimental data. Radiation modelling was performed with the discrete transfer method. In the 1995 study, computations were validated against measurements of gas temperature, species concentrations and wall heat flux for standard operating conditions, partial load operation and low excess air conditions. Although occasionally only qualitative agreement between theory and experimental results was found, the general conclusion was that the models were extremely useful in the analysis of utility boiler heat transfer performance.

The increasing sophistication of combustion chemistry models within the CFD codes has led to their application to the problem of the prediction of pollutants and emissions. Industrial glass furnaces operate at particularly high temperatures, where thermal NO formation and consequent emissions become an important problem. Correct prediction of the temperature, $\mathrm{O}_{2}$-concentration and velocity field is of paramount importance. Carvalho et al (1990, 1992a) reported early studies of this problem using the time-averaged conservation equations in conjunction with the k- epsilon turbulence model. The thermal radiation was calculated using the discrete transfer method. The chemical reactions associated with the heat release were assumed to be fast. The fluctuations of the scalar properties were accounted for by the use of a clipped-Gaussian probability density function. The Zeldovich mechanism was incorporated in the model in order to predict instantaneous formation rates of thermal NO from atmospheric nitrogen. Detailed validation was not possible, due to the lack of data, but parametric studies demonstrated the ability of the model to evaluate the effects of combustion excess-air level, air preheating, and fuel composition on the nitric oxide emissions on NO emissions of a cross-fired regenerative furnace.

Carvalho et al (1992b) reported a study of a model of a cement kiln, which employs a version of the discrete transfer method implemented for a three-dimensional cylindrical enclosures was used. Direct comparison of the computed results with experimental data was not possible, but the overall heat fluxes that were calculated were typical of those found in dry-process cement kilns. Comparison of the results for enclosures containing scattering and non-scattering media highlighted the need for a proper account of the scattering properties of the medium in this case. 
The above developments have progressively led to the ability to predict emissions from pulverised coal furnaces. Along with the models of combustion chemistry the radiation models are required to account for absorption, emission and scattering in the medium. Coimbra et al (1994) present the results of a $\mathrm{NO}_{\mathrm{x}}$ formation model applied to a $300 \mathrm{MWe}$ front-wall fired boiler under different operating conditions and report good agreement of measured $\mathrm{NO}_{\mathrm{x}}$ emissions in the flue gases. Visona and Stanmore (1996) modelled $\mathrm{NO}_{\mathrm{x}}$ formation in a frontwall-fired 275 MWe utility boiler. They examined the effectiveness of $\mathrm{NO}_{\mathrm{x}}$ mitigation by means of different firing patterns, which included: burners out of service, overfired air and excess air. Combustion and modelling studies were carried out with two coals of different fuel-nitrogen content. A sensitivity analysis was included, to test the relative importance of selected $\mathrm{NO}_{\mathrm{x}}$ formation chemistry modelling parameters. The study found that reasonable predictions were possible with a simplified $\mathrm{NO}_{\mathrm{x}}$ chemistry combined with a relatively coarse computational grid. The predicted furnace exit NO concentrations differed by $0-30 \%$ from those measured.

The study of Adams and Smith (1992) used the discrete ordinates method to model radiative heat transfer in two industrial scale furnaces. Radiative heat flux predictions in a gas furnace with cooling pipes in the combustion chamber were shown to be in good agreement with the experimental data. The application of the discrete ordinates method in a coal fired utility boiler showed good agreement only in a part of the geometry and the flame zone; radiative heat transfer was not well predicted. Adams and Smith (1994) have also employed the discrete ordinates method to study the modelling of soot and turbulence-radiation coupling in an industrial furnace. No experimental validation was provided but the results obtained showed the expected trends. The application of the recent finite volume method of Raithby and Chui (1990) to furnace type problems was demonstrated by Chui et al (1993). In their study a coupled coal combustion model with the finite volume radiation technique has shown to produce good agreement with experimentally measured net heat transfer to the furnace walls.

The accurate modelling of radiative heat transfer in furnaces now enables detailed examination of pollutant formation mechanisms. For example, Yuan et al (1997) investigate the role played by soot and oil cenosphere formation on heat transfer, boiler fouling and emissions for a threedimensional oil-fired industrial-type furnace. The flow is solved on non-uniform $40 \times 44 \times 45$ and $60 \times 66 \times 67$ grids, the radiation problem was solved with the discrete transfer method using a $12 \times 22 \times 18$ mesh. 
The Monte Carlo method has also been used in a number of situations involving combustion to calculate radiative heat transfer. A more complete discussion of Monte Carlo techniques and their applications can be found in Howell (1998). Stewart and Cannon (1971), Vercammen and Froment (1980), Kudo et al (1995) are among many studies which have used Monte Carlo techniques to predict heat transfer in practical combustor systems. The method has been shown to be robust and accurate in predicting heat transfer in combusting problems.

Stuttaford and Rubini (1998) applied an integrated CFD/radiation model and a network model to study the performance of a gas turbine combustor. Radiation represents the most difficult mode of heat transfer to simulate in the combustor environment. A novel variation of the discrete transfer radiation model was presented and validated for use within the network solver. The effect of the representation of the wall liner boundary condition on the prediction of liner temperature was evaluated in an annular gas turbine combustor at a typical high-power operating condition.

In most of the integrating studies reviewed above, the temperature dependence of the properties of the medium was represented by means of Hottel and Sarofim’s “two-mixed-grey-and-oneclear” gas formulation assuming that $\mathrm{CO}_{2}$ and $\mathrm{H}_{2} \mathrm{O}$ are the only participating gas constituents. More sophisticated, and yet economical, descriptions of participating media are under development. Docherty and Fairweather (1988) describe an implementation of the discrete transfer method derived from integration of the spectral equation of radiative transfer. The exponential wide-band model is used and validated for mixtures of $\mathrm{CO}_{2}, \mathrm{H}_{2} \mathrm{O}$ and soot by comparison with results from a narrow band statistical model of radiative transfer. Bressloff et al (1996) present a coupled model for the prediction of soot, gas species concentrations and radiative exchange in turbulent combustion applied to the case of a confined, turbulent, jet diffusion flame burning methane in air. The slow soot formation processes are described by a model that is coupled here to the discrete transfer method, incorporating a weighted sum of grey gases (WSGG) - which provides a better description of the nongrey radiative properties of combustion media - solution to the radiative transfer equation. Good agreement is reported between numerical results and experimental measurements of mixture fraction, temperature and soot volume fraction. A more detailed discussion on the calculation of radiative properties in combustion applications can be found in Carvalho and Farias (1998).

Recent work at Loughborough University has focused on the application of radiation models to the prediction of the performance of IC engines. The discrete transfer method has been 
successfully applied to predict radiative heat transfer from diesel and petrol engines and the results generally agree well with experimental trends (Blunsdon et al, 1992, 1993). In other applications Blunsdon et al (1996), Beeri et al (1996) have used a modified version of the CFD code KIVA-II to model the transient behaviour of buoyant turbulent diffusion flames and of high-momentum vertical and horizontal flames burning in still air and in ambient air flows. Accurate calculation of radiative transfer has enabled the modelling of the presence and frequency of large-scale pulsations. Axial distributions of temperature, velocity, and chemical species concentrations in turbulent buoyant diffusion flames were predicted with good accuracy. The radial structure of the flame was less well represented. The axial radiative heat flux distribution from the flame highlighted deficiencies in the soot generation model, suggesting that a model of soot particle growth was required. Predictions of temperature and flame profiles in the high momentum flames were found to be in good agreement with data, but predictions of radiative heat transfer were not entirely satisfactory. This was attributed to uncertainties in the measurements and limitations on the number of rays that could be used.

Most recently, Henson (1998) has integrated a discrete transfer/generalised WSGG model into the KIVA-II code for three-dimensional, full-cycle engine calculations. The WSGG model used is able to economically capture the spectral nature of an arbitrary gas mixture. Compared with less general, correlated approaches this results in better predictions of the peak radiative heat flux, and its variation, at the cylinder walls. Some sample results will be presented later.

Other prominent applications of radiation modelling in the context of combusting systems include those in the field of fire safety. Early studies of Lockwood and Malalasekera (1988, 1991) and more recent work by Lewis et al (1997) have used the discrete transfer method to model fire behaviour in compartments. Fairweather et al (1992) predicted the flame structure and thermal radiation around a turbulent reacting jet discharging into a cross-flow in the context of gas flaring operations. Radiation only represents about $20 \%$ of the total heat loss and the received radiation levels from the flame at a transducer could be post-processed outside the main CFD procedure. For fully converged results it was necessary to employ 1024 rays in the discrete transfer method. Further developments of this model include Cook et al (1997) and Baillie et al (1998), who model jet fires due to sonic releases of natural gas and the effect of their impingement on a pressure-vessel. Tryfonopoulos et al (1995) give a model, which examines scenarios of real-time development of a forest fire in complex topography. Thermal radiation is calculated by the discrete transfer method. The role played by convective and radiative effects on 
forest fire propagation is investigated and their relative importance is assessed for several ambient wind speeds.

\section{METHODS FOR COMPLEX GEOMETRIES AND RECENT ADVANCES}

The Monte Carlo method is a statistical method and to achieve an accurate solution a large number of energy bundles have to be fired in the calculation process and a number of repeated calculations have to be performed to get an estimation of the error of the solution. It is applicable in any geometrical configuration and capable of handling isotropic, anisotropic and non-grey problems. Monte Carlo solutions are widely used as benchmarks to evaluate the performance of other radiation calculation methods in complex geometry applications, Parthasarathy et al (1995). The method is computationally expensive, but considerable speed up can be achieved by incorporating an efficient ray tracing technique such as the method described in Henson and Malalasekera (1997a).

The discrete transfer method is mathematically simple and also applicable to any geometrical configuration. When a fast and efficient ray tracing algorithm is used the method is computationally efficient. It is well established in application to combustion related problems and extensions of the method to isotropic scattering problems and non-grey calculations have been demonstrated in Carvalho et al (1991), Henson and Malalasekera (1997b). The method is, however, not suitable for anisotropic scattering applications. Several enhancements and modifications to the original method have been proposed, e.g. Cumber (1995) and Coelho and Carvalho (1997). Our predictive experience is that when an adequately refined mesh and a sufficiently large number of rays are used the standard method produces results which are comparable in accuracy to Monte Carlo solutions. The accuracy of the method depends on two factors, surface discretisation (number of surface elements used to fire rays) and angular discretisation (number of rays used per point). Mathematical expression for the errors resulting from these discretisation practices have been derived for simple situations (Versteeg et al, 1999a $\& \mathrm{~b})$. The studies using transparent media show that the decay rate of the angular discretisation error $\varepsilon_{H}$ with increasing ray number depends on the smoothness of the irradiation. For smooth irradiating intensity $\varepsilon_{H} \propto 1 / N_{R}$, for piecewise sources $\varepsilon_{H} \propto 1 / \sqrt{N_{R}}$ and for intensity fields field with derivative discontinuities $\varepsilon_{H} \propto 1 / N_{R}$. The surface discretisation error is generally small compared to $\varepsilon_{H}$ and can be reduced by refinement of the surface mesh. 
In the YIX method integrations are carried out by using special integration functions. The method uses the $S_{N}$ approximation for angular discretisation. The accuracy, therefore, depends on the $S_{N}$ order. Like the discrete transfer method, the YIX method is also based on ray tracing. However, the computational resource requirements are higher for the YIX method than the discrete transfer method, since the YIX method requires ray firing from the points inside the medium as well as points on surface elements. Applications involving isotropic and anisotropic scattering situations and nongrey media and arbitrary shaped geometries have been demonstrated in the literature (Hsu et al, 1992, Hsu and Farmer, 1995) The method is relatively new and applications involving combustion calculations are not known to the authors. As the method is capable of handling anisotropic scattering it could be used in cases where the discrete transfer method cannot be used. Theoretical and numerical comparisons of the YIX method with the discrete transfer method will be reported in Henson and Malalasekera (1999).

The standard discrete ordinates method is one of the most widely used methods for simple geometrical situations among combustion researchers. Its computational efficiency is a major advantage of the method. The original method has been extensively evaluated over the years for numerous types of radiative heat transfer problems including isotropic, anistropic and non-grey problems. The results obtained by the method are sensitive to the value of $\gamma$ in Eqs. (35) and (36) and in some cases negative unphysical intensities may result. Fiveland has shown that such negative intensities may be minimised (but not totally avoided) if finite volume dimensions are appropriately selected, Fiveland (1988) and see also Modest (1993). The accuracy of the method depends on the order of the approximation. Low order $S_{N}$ solutions suffer from the errors known as 'ray effects' and the solution also depends on the spatial interpolation scheme used. The effect of these phenomena on accuracy and predictive capabilities of the $\mathrm{S}_{\mathrm{N}}$ method has been studied by Chai et al (1993), Coelho et al (1995a) and Selçuk and Kayakol (1996). Variable and exponential differencing schemes have been developed to avoid overshoots, undershoots and physically unrealistic results arising from spatial differencing practices. More advanced and accurate differencing schemes are available in the literature, see e.g. Chai et al (1994b), Jessee and Fiveland (1996) and Liu et al (1996).

The application of the standard discrete ordinates method to non-orthogonal body fitted coordinates systems is not straightforward. In these cases the method requires special formulations that are much more mathematically complex than the original one. Sakami et al $(1996,1998)$ and Charette et al. (1997) have developed a new discrete ordinates algorithm for two- and three- 
dimensional arbitrary geometries. The method uses triangular (in 2D) and tetrahedral (in 3D) cells and is therefore readily applicable to unstructured mesh systems often used in modern CFD codes. The basic equations are derived by integrating the RTE over a control volume. The required average intensities over the faces of a tetrahedral cell are obtained by numerical integration of the characteristic equations. The method appears to be robust and applicable to any complex geometry configurations and its ability to handle tetrahedral mesh structures without additional modifications is appealing to unstructured CFD codes. Other formulations of the discrete ordinates method to complex geometry situations have been reported in Beak and Kim (1997, 1998).

The newly developed finite volume based techniques are applicable to arbitrary shaped complex geometrical configurations. They have been shown to be capable of solving problems involving isotropic and anisotropic scattering problems (Chai and Modar, 1996, Beck et al, 1998, Murthy and Mathur, 1998). The application of the finite volume method in a practical combustor geometry has also been demonstrated by Chui et al (1993). Further applications involving a wide range of combustion-related problems and more extensive benchmark comparison are required to access the capabilities and efficiency of these new techniques.

\section{Comparison of Calculation Methods}

Figure 1 shows a comparison of all methods described above for a bench-mark problem which has been widely used in radiative heat transfer literature to evaluate calculation methods. The configuration is the IFRF furnace geometry outlined in the paper by Jamaluddin and Smith (1988). In our calculations all the techniques outlined above are used to solve the problem described as the IFRF-M3 Trial (Flame 10). The furnace dimensions are 6.0x2.0x2.0 $\mathrm{m}$ in $x, y$ and $z$ directions and the measured temperatures by Hyde and Truelove (1977) are used to define the problem, other essential details can be found in Jamaluddin and Smith (1988). The discrete ordinates method used here is the standard discrete ordinates method while Monte Carlo, YIX and discrete transfer methods are used in their most general form (i.e. applicable to any geometry). The results obtained by the Monte Carlo method here are considered to be the most accurate solution and computing resources used in other methods were increased until comparable results with the Monte Carlo solution were obtained. The discrete transfer calculation has been performed with 400 rays per point, while $S_{16}$ quadrature is used for the discrete ordinates and YIX angular discretisation. 
Figure 1 shows the calculated incident radiative flux on the floor and the roof of the furnace and Figure 2 shows the distribution of net radiative heat fluxes on the surfaces of the furnace floor (note the top surface shown in Figure 2 is the floor). The grid used for the calculation is also shown in Figure 2. It can be seen from the Figure 1 that all solutions compare well with the Monte Carlo solution and the differences are very small. A closer look at the actual numerical values (not presented here) reveals that discrete ordinate solution has the highest deviation. This could be attributed to its spatial differencing practice and the accuracy of the quadrature set. Figure 3 shows a comparison of low order solutions $S_{6}, S_{8}$ and $S_{12}$ with $S_{16}$. Wiggles of the solution are clearly more noticeable for lower order results.

A further comparison of methods in a more complex L-shaped geometry (see Figure 4), which can be modelled using a body fitted coordinates system, is given in Figure 5. The problem specification is: (i) all walls black at $500 \mathrm{~K}$ and (ii) the enclosure is filled with an emitting/absorbing and non-scattering medium at a temperature of $1000 \mathrm{~K}$. Predicted net radiative heat flux on line B-B (Figure 4) for different absorption coefficients $\kappa=0.5,1.0,2.0$, 5.0 and 10.0 using Monte Carlo, DTM, discrete ordinates method and the YIX method is shown in Figure 5. The discrete transfer solution is from Malalasekera and James (1995). Discrete Ordinates solution is from Sakami et al (1998). The Monte Carlo results are by Maltby (1996) and the YIX solution by Hsu and Tan (1996). It can be seen that results of all methods agree well. The conclusion that can be drawn is that when adequate resources are used all methods produce equally good results that are in agreement with the Monte Carlo solution.

Finally, we present some sample results from flow-radiation calculations of a spark ignition engine Henson (1998). In this simulation the turbulent flow and combustion were simulated using the engine CFD code KIVA-II. A discrete transfer/generalised WSGG model was used to find the radiant losses to the cylinder walls from the high-temperature combustion products. Figure 6 shows a series of snap-shots plotting the growth, and subsequent decline, in the instantaneous wall radiative heat flux at selected crank positions during the combustion phase. Also shown is the body-fitted nonorthogonal mesh used to describe the complex pentroof combustion chamber geometry for both flow and radiation calculations. The study is an excellent demonstration of the radiation model's ability to cope with complex geometry and strong transient property gradients in a combined mode analysis. 


\section{MODELLING OF TURBULENCE/RADIATION INTERACTIONS IN COMBUSTION}

Most combustion equipment operates under highly turbulent conditions and radiative heat transfer is affected by turbulent combustion. Due to complexity of modelling radiative heat transfer, current radiation calculation practice takes into account only the mean temperature and species fields and ignores their turbulent fluctuations. Spectral and time-resolved measurements in a model gas turbine combustor by Koch et al (1994) have shown highly fluctuating intensities resulting from fluctuations in both temperature and the concentrations of radiating species; it is therefore important to include these fluctuations in radiation calculations. A small number of studies have attempted to develop modelling techniques to include turbulence effects in radiation calculations; these include the work by Song and Viskanta (1986), Kounalakis et al (1981) and Chan et al (1994). Some of these published techniques are mathematically complex and not readily applicable in engineering calculation involving complex geometries. At Loughborough we have attempted to develop a simple and more useable technique to incorporate turbulence effects. We have carried out several path-length based studies to demonstrate the effect of inclusion and non-inclusion of fluctuating temperature and property effects in radiative transfer

Figure 7(a) shows a detailed quasi-transient calculations along a path-length in a medium $\left(E_{b}\right)$ where temperatures and properties are allowed to randomly fluctuate (up to $\pm 25 \%$ ) about their mean values. The curve marked (2) shows the fluctuating flux calculated using the discrete transfer method in a quasi-transient manner using instantaneous temperature and property values. Solid circles are Monte Carlo results. The curve marked (1) is the result from a calculation using local average temperature and properties. The curve marked (3) is the mean of the fluctuating flux result. As can be seen, there is a clear difference between the curve (1) and curve (3) which highlights the difference between inclusion and non-inclusion of fluctuations in the calculation.

It is not practical to perform quasi-transient calculations in a general-purpose CFD procedure to resolve turbulence fluctuations. In order to develop a practical alternative we have conducted parametric studies to establish the individual fluctuating effects of all parameters involved in the transfer equation. It was established that temperature, through its fourth power dependency, is the most significant parameter and compared to the effect produced by temperature fluctuations the effect produced due to grey absorption coefficient fluctuations is small. This allows a considerable simplification in the time-averaging of the transfer equation. For emitting absorbing 
and non-scattering media we have developed the following approximate discrete transfer formulation to include temperature fluctuations.

$$
\bar{I}_{n+1}=\bar{I}_{n} e^{-\kappa s}+\bar{I}_{b}\left(1-e^{-\kappa s}\right)
$$

where $\bar{I}_{b}=\frac{\sigma}{\pi}\left[\bar{T}^{4}+6 \bar{T}^{2} \overline{t^{\prime 2}}+\overline{t^{\prime 4}}\right]$

When this equation is used to perform the same calculation using local fluctuations along the path it results in the same curve as (3) (in Fig. 7(a)) indicating that the technique is accurate enough to reproduce fluctuating effects. We have further explored this approach in predicting radiation from an unconfined methane diffusion flame studied by Jeng and Faeth (1984). The burner diameter was $5 \mathrm{~mm}$ and the flow conditions involved $\mathrm{Re}=11700$ using $96 \%$ methane by volume. Predictions of this flame were attempted by using a 2D grid of 120x60 cells in the CFD calculations, combustion model using a $\beta$ pdf and the modified DT method to simulate radiation fluctuations. Results are shown in Figure 7(b)-(d). Figure 7(b), shows the predicted temperature field and comparison of calculated and measured spectral radiation intensities at two axial locations agree well. Figure 7(c) shows the radial temperature profile at $\mathrm{x} / \mathrm{D}=52.5$ and maxima and minima of temperature fluctuations predicted by the pdf combustion model are also shown. The intermediate values have been randomly regenerated to use in the simulation. The temperature fluctuations here are more representative and correlated than those used in Figure 7(a). Figure 7(d) shows the result of path length based DT approach applied to a line of sight at axial location $\mathrm{x} / \mathrm{D}=52.5$. RADCAL was used for property calculations. It can be seen that the $\mathrm{DT}$ approach is able to reproduce the true mean of the fluctuating flux distribution.

\section{SUMMARY}

The Monte Carlo method, discrete transfer method, the YIX method and the discrete ordinates method which have been discussed in this paper are applicable with modern day CFD finite volume computational procedures which use non-orthogonal body-fitted mesh systems. The discrete ordinates method does not require ray tracing, which is a common feature in all other techniques. However, to get similar accuracy with other techniques higher order discrete ordinates sets are required with the discrete ordinates method. The Monte Carlo method requires very substantial computer resources and cpu time, but produces accurate benchmark results. The other methods require similar, but smaller, computer resources when applied to the same problem to achieve accuracy that is similar to the Monte Carlo method. Their performance, as well as that of the Monte Carlo method, depends on the efficiency of the ray tracing algorithm. In this paper we have reviewed some recent and notable application of radiative heat transfer 
techniques and comparative results were presented for the most useful methods. An example application in a complex geometry was presented to illustrate the capability of the discrete transfer method. Inclusion of turbulence effects in radiation calculation is important in highly turbulent combustion systems. A simple technique that is capable of handling fluctuating effects along a path length was described, demonstrating that radiative heat transfer methods based on ray tracing techniques can be easily adopted to include temperature fluctuations from pdf based combustion models.

\section{ACKNOWLEDGEMENT}

The authors wish to gratefully acknowledge the financial support received from the Engineering and Physical Research Council (EPSRC) and the Defence Evaluation and Research Agency (DERA) of the UK for a part of the work reported in this paper.

\section{NOMENCLATURE}
A area of a surface element $\left[\mathrm{m}^{2}\right]$
$E_{g} \quad$ gas blackbody emissive power $\left[\mathrm{W} / \mathrm{m}^{2}\right]$
$E_{s} \quad$ surface blackbody emissive power $\left[\mathrm{W} / \mathrm{m}^{2}\right]$
$I \quad$ intensity of radiation $\left[\mathrm{W} / \mathrm{m}^{2} \mathrm{sr}\right]$
$Q_{g} \quad$ net radiative heat source in a volume [W]
$Q_{s} \quad$ net radiative heat flow on a surface [W]
$R \quad$ random number from uniform distribution [-]
$S \quad$ radiative source function $\left[\mathrm{W} / \mathrm{m}^{2} \mathrm{sr}\right]$
$q_{-} \quad$ incident flux onto a surface $\left[\mathrm{W} / \mathrm{m}^{2}\right]$
$q_{+} \quad$ emitted flux from a surface $\left[\mathrm{W} / \mathrm{m}^{2}\right]$
q flux vector
r position vector
$s \quad$ geometric path length [m]
$\delta s \quad$ length of path segment $[\mathrm{m}]$
$\hat{\mathbf{s}} \quad$ unit vector in a given direction [-]
$\mathrm{V}$ volume $\left[\mathrm{m}^{3}\right]$
$x, y, z$ Cartesian coordinates [m]
$\beta$ extinction coefficient $\left[\mathrm{m}^{-1}\right]$
$\varepsilon \quad$ emissivity [-] 


$$
\begin{array}{ll}
\kappa & \text { absorption coefficient }\left[\mathrm{m}^{-1}\right] \\
\sigma & \text { scattering coefficient }\left[\mathrm{m}^{-1}\right] \\
\Phi & \text { scattering phase function, [-] } \\
\psi & \text { bilinear shape function [-] } \\
\omega & \text { single scattering albedo [-] } \\
\Omega & \text { solid angle [sr] }
\end{array}
$$

All other symbols are defined in the text.

\section{REFERENCES}

Abbas A.S., Lockwood F.C., Salooja A.P. (1984), Prediction of the Combustion and Heat Transfer Performance of a Refinery Heater, Comb. Flame, Nov 1984, Vol.58, No.2, pp. 91101.

Adams, B.R. and Smith, P.J., (1992), Three-dimensional Discrete Ordinates Modelling of Radiative Transfer in Industrial-Scale Furnaces, HTD Vol. 203, pp. 137-144.

Adams, B.R. and Smith, P.J., (1994), Modelling Effects of Soot and Turbulence-Radiation Coupling on Radiative Transfer in an Industrial Furnace, HTD Vol. 276, pp. 177-190.

Baek, S.W. and Kim, M.Y., (1997) Modification of the Discrete Ordinates Method in an Axysysmmetric Cylindrical Geometry, Num. Heat Transfer, Part B, Vol. 31, No. 3, pp. 313-326.

Baek, S.W. and Kim, M.Y., (1998), Radiative Heat Transfer in a Body-Fitted Axisymmetric Cylindrical Enclosure, J. Thermophys. Heat Transfer, Vol. 12, No. 4, pp. 596-599.

Baek, S.W., Kim, M.Y. and Kim, J.S., (1998), Nonorthogonal Finite-Volume Solutions of Radiative Heat Transfer in a Three-Dimensional Enclosure, Num. Heat Transfer, Part B, Vol. 34, No. 4, pp. 419-437.

Baillie, S., Caulfield, M., Cook, D.K. and Docherty, P. (1998), Phenomenological Model for Predicting the Thermal Loading to a Cylindrical Vessel Impacted by High Pressure Natural Gas Jet Fires, Process Safety and Environmental Protection: Trans. IChemE, Part B, Vol.76, No.1, pp. 3-13.

Beeri, Z., Blunsdon, C.A., Malalasekera, W. and Dent, J.C. (1996) Comprehensive Modelling of Turbulent Flames with the Coherent Flame-sheet Model - Part II: High-momentum Reactive Jets, J. Energy Resources Technology, Vol.118, No.1, pp. 72-76. 
Blunsdon, C.A., Malalasekera, W. and Dent, J.C. (1992) Application of the Discrete Transfer Model of Thermal Radiation in CFD Simulation of Diesel Engine Combustion and Heat Transfer, SAE Paper Number 922305.

Blunsdon, C.A., Malalasekera, W. and Dent, J.C. (1993) Modelling Infrared Radiation from the Combustion Products in a SI Engine, SAE Paper Number 932699.

Blunsdon, C.A., Beeri, Z., Malalasekera, W. and Dent, J.C. (1996) Comprehensive Modelling of Turbulent Flames with the Coherent Flame-sheet Model - Part I: Buoyant Diffusion Flames, J. Energy Resources Technology, Vol.118, No.1, pp. 65-71.

Boyd, R.K and Kent, J.H., (1986), Three-Dimensional Furnace Computer Modelling, Twentyfirst Symp. (Int.) on Combustion, The Combustion Institute, pp. 265-274.

Bressloff, N.W., Moss, J.B. and Rubini, P.A., (1996a) Assessment of a Differential Total Absorptivity Solution to the Radiative Transfer Equation as Applied in the Discrete Transfer Radiation Model, Num. Heat Transfer, Part B, Vol. 29, No. 3, pp. 381-397.

Bressloff, N.W., Moss J.B. and Rubini P.A. (1996b), CFD Prediction of Coupled Radiation Heat Transfer and Soot Production in Turbulent Flames, Twentysixeth Symp. (Int.) on Combustion, Part 2 (of 2), Napoli, Italy, pp. 2379- 2386.

Carvalho, M.G. and Coelho, P.J. (1990), Numerical Prediction of an Oil-fired Water Tube Boiler, Engineering Computations, Vol.7, No.3, pp. 227-234.

Carvalho, M.G., Semiao, V., Lockwood F.C. and Papadopoulos C. (1990), Predictions of Nitric Oxide Emissions from an Industrial Glass- Melting Furnace, J. Inst. Energy, Vol.63, No.454, pp. 39-47.

Carvalho, M.G., Farias, T. and Fontes, P. (1991), Predicting Radiative Heat Transfer in Absorbing, Emitting, and Scattering Media using the Discrete Transfer Method, ASME FEDVol. 160, pp. 17-26.

Carvalho, M.G., Semiao, V.S. and Coelho, P.J. (1992a), Modelling and Optimization of the NO Formation in an Industrial Glass Furnace, J. Engineering for Industry, Vol.114, No.4, pp. 514-523.

Carvalho, M.G., Farias, T. and Martins, A. (1992b), Three-dimensional Modelling of the Radiative Heat Transfer in a Cement Kiln, Proc. Second Int. Conf. Advanced Computational Methods in Heat Transfer: Forced Convection and Combustion Simulation, Milan, Italy, pp. 141-160.

Carvalho, M.G. and Farias, T.L., (1998), Modelling of Heat Transfer in Radiating and Combustion Systems, J. Chemical Engineering Research and Design, Trans IChemE, Vol. 76, Part A, pp. 175-184. 
Chai, J.C., Lee, H.S. and Patankar, S.V., (1993), Ray Effect and False Scattering in the Discrete Ordinates Method, Num. Heat Transfer, Part B, Vol. 24, pp. 373-389.

Chai, J. C., Lee, H.S. and Patankar, S.V., (1994a), Finite-Volume Method for Radiation Heat Transfer, J. Thermophys. Heat Transfer, Vol., 8, No. 3, pp 419-425.

Chai, J.C., Parthasarathy, G., Lee, H.S. and Patankar, S. V., (1994b), Evaluation Spatial Differencing Practices for the Discrete Ordinates Method, J. Thermophys. Heat Transfer, Vol. 8, No. 1, pp. 140-144.

Chai, J.C., Parthasarathy, G., Lee, H.S. and Patankar, S. V., (1994c), Finite-Volume Radiation Heat Transfer Procedure for Irregular Geometries, J Thermophys. Heat Transfer, Vol. 9, No. 3, pp. 410-415.

Chai J.C and Modar, J.P. (1996), Spatial-Multiblock Procedure for Radiation Heat Transfer, ASME HTD-Vol 332, pp. 119-127.

Chan, S.H., (1987), Numerical Methods for Multidimensional Radiative Transfer Analysis in Participating Media, Ann. Rev. Num. Fluid Mech. Heat Transfer, V1, pp. 305-350.

Chan, S.H., Pan, X.C. and Zhang, J., (1994), A Two-dimensional Non-stationary Causal Stochastic Model for Turbulence/Radiation Interaction in Flames, Twenty-fifth Symp. (Int.) on Combustion, pp.1115-1123.

Chandrasekhar, S., (1960), Radiative Transfer, Dover Publications.

Charette, A, Sakami, M. and Le Dez, V., (1997), Analysis of Radiative Heat Transfer in Enclosures of Complex Geometry using the Discrete Ordinates Method, Radiative Heat Transfer-II, Mengüç, M.P. (Ed.), Proc. Second Int. Symp. on Radiation Transfer, Kusadasi, Turkey, Begell House Inc., pp 253-270.

Chui, E.H., Raithby, G.D and Hughes, P.M., (1992), Prediction of Radiative Transfer in Cylindrical Enclosures with the Finite Volume Method, J. Thermophys. Heat Transfer, Vol. 6, No. 4, pp. 605-611.

Chui, E.H. and Raithby, G.D., (1993), Computation of Radiative Heat Transfer on a Nonorthogonal Mesh Using the Finite Volume Method, Num. Heat Transfer, Part B, Vol. 23, pp. 269-288.

Chui, E.H.,Hughes, P.M, and Raithby, G.D., (1993), Implementation of the Finite Volume Method for Calculating Radiative Transfer in a Pulverised Fuel Flame, Combust. Sci. and Tech., Vol. 92, pp. 225-242.

Coelho, P.J., Goncalves, J.M., and Carvalho, M.G., (1995a), A Comparative Study of Radiation Models for Coupled Fluid Flow/Heat Transfer Problems, Proc. 9th Int. Conf. for Num. Methods in Thermal Problems, Lewis, R.W., and Dubertaki, P. (Eds), Pineridge Press, Swansea, Vol. IX, part 1, pp. 378-389. 
Coelho, P.J. and Carvalho, M.G. (1995b), Evaluation of a Three-dimensional Model for the Prediction of Heat Transfer in Power Station Boilers, Int. J. Energy Research, Vol.19, No.7, pp. 579-592.

Coelho, P.J. and Carvalho, M.G., (1997), A Conservative Formulation of the Discrete Transfer Method, J. Heat Transfer, Vol. 115, pp. 486-489.

Coimbra C.F.M., Azevedo J.L.T., Carvalho M.G. (1994), 3-D Numerical Model for Predicting NOx Emissions from an Industrial Pulverised Coal Combustor, Fuel, Vol.73, No.7, pp. 11281134.

Cook, D.K., Cumber, P.S., Fairweather, M. and Shemirani, F. (1997), Modelling Free and Impacting Underexpanded Jet Fires, Proc. 1997 Symp. on Hazards XIII Process Safety - The Future, Manchester, UK, Apr 22-24 1997, Inst. Chem. Engrs. Symp. Series, No.141, pp. 127138.

Cumber, P.S. (1995), Improvements to the Discrete Transfer Method of Calculating Radiative Heat Transfer, Int. J. Heat Mass Trans., Vol. 38, No. 3, pp. 2251-2258.

Denison, M. K. and Webb, B.W., (1993), A Spectral Line Based Weighted-sum-of-grey-gases Model Arbitrary RTE Solvers, J. Heat Transfer, Vol. 115, pp. 1004-1012.

Denison, M.K. and Webb, B.W., (1995), The Spectral Line Based Weighted-sum-of-grey-gases Model in Non-isothermal Non-homogeneous Media, J. Heat Transfer, Vol. 177, pp. 359-365. Docherty, P. and Fairweather, M., (1988), Predictions of Radiative Transfer from Nonhomogeneous Combustion Products using the Discrete Transfer Method, Comb. Flame, Vol.71, No.1, pp. 79-87.

Edwards, D.K. and Balakrishnan, A. (1973), Thermal Radiation by Combustion Gases, Int. J. Heat Mass Transfer, 16, pp 25-40.

Fairweather, M., Jones, W.P. and Lindstedt, R.P. (1992), Predictions of Radiative Transfer from a Turbulent Reacting Jet in a Cross-wind, Comb. Flame, Vol.89, No.1, pp. 45-63.

Farmer, J.T., (1995), Improved Algorithms for Monte Carlo Analysis of Radiative Heat Transfer in Complex Participating Media, Ph.D. Thesis, The University of Texas at Austin, Texas, USA. Fiveland, W.A., (1982), A Discrete Ordinates Method for Predicting Radiative Heat Transfer in Axisymmetric Enclosures, ASME Paper 82-HT-20.

Fiveland, W.A., (1988), Three-Dimensional Radiative Heat Transfer Solutions by the DiscreteOrdinates Method, J. Thermophys. Heat Transfer, Vol. 2, No. 4, pp. 309-316.

Fiveland, W.A., (1991), The Selection of Discrete Ordinates Quadrature Sets for Anisotropic Scattering, ASME HTD Vol. 160, pp. 89-96. 
Fiveland, W.A. and Jessee, J. P., (1994), Comparisons of Discrete Ordinate Formulations for Radiative Heat Transfer in Multidimensional Geometries, Radiative Heat Transfer: Current Research, ASME HTD-Vol 276, pp. 49-57.

Henson, J.C. and Malalasekera, W. (1997a) Comparison of Discrete Transfer and Monte Carlo Methods for Radiative Heat Transfer in Three-Dimensional Non-Homogeneous Scattering Media, Num. Heat Transfer Part A: Applications, Vol.32 No. 1, pp. 19-36.

Henson, J.C. and Malalasekera, W., (1997b) Benchmark Comparisons with Discrete Transfer Method Solutions for Radiative Heat Transfer in Three-dimensional, Nongrey, Scattering Media, Radiative Heat Transfer-II, Mengüç, M.P. (Ed.), Proc. Second Int. Symp. on Radiation Transfer, Kusadasi, Turkey, Begell House Inc., pp. 195-207.

Henson J.C. (1998), Numerical Simulation of S.I. Engines with Special Emphasis on Radiative Heat Transfer, Ph.D. Thesis, Loughborough University, Loughborough, UK.

Henson, J.C. and Malalasekera, W., (1999), An Evaluation of the Discrete Transfer and YIX Radiation Methods for Complex Participating Media, to be presented at the 33rd National Heat Transfer Conference, Albuquerque, New Mexico. ASME paper no. HTD99-5129.

Howell, J.R and Perlmutter, M., (1964), Monte Carlo Solution of Thermal Transfer in Nongrey Non-isothermal Gas with Temperature Dependent Properties, AIChE Journal, Vol. 10, No. 4, pp. 562-567.

Howell, J.R., (1988), Thermal Radiation in Participating Media: the Past, the Present and Some Possible Future, J. Heat Transfer, Vol. 110, pp. 1220-1226.

Howell, J.R., (1998), The Monte Carlo Method in Radiative Heat Transfer, J. Heat Transfer, Vol. 120, pp. 547-560.

Hsieh, T.C. and Greif, R., (1972), Theoretical Determination of the Absorption Coefficient and the Total Band Absorptance Including a Specific Application to Carbon Monoxide, Int. J. Heat Mass Transfer, Vol. 15, pp. 1477-1487.

Hsu, P.F., Tan, Z and Howell, J.R., (1992), Application of the YIX Method to Radiative Heat Transfer within a Mixture of Highly Anisotropic Scattering Particulate and Non-grey Gas, 28th ASME National Heat Transfer Conference, Aug 1992, ASME HTD-Vol 203, pp. 285-300.

Hsu, P., Tan, Z. and Howell, J. R.,(1993), Radiative Transfer by the YIX Method in Nonhomogeneous, Scattering, and Nongrey Media, J. Thermophys. Heat Transfer, Vol. 7, No. 3, pp. 487-495.

Hsu P.F and Farmer, J.F. (1995), Benchmark Solutions of Radiative Heat Transfer within Nonhomogeneous Participating Media Using the Monte Carlo and YIX Methods, ASME HTDVol. 315, pp. 29-36. 
Hsu P.F and Tan, Z. (1996), The Radiative and Combined Mode Heat Transfer within the LShaped Nonhomogeneous and Nongrey Participating Media, ASME HTD-Vol. 325, pp 13-24. Hyde, D.J. and Truelove, J.S., (1977), The Discrete Ordinates Approximation for Multidimensional Radiant Heat Transfer in Furnaces, Technical report, UKAEA Report No. AERE-R-8502, AERE Harwell, Oxfordshire, UK.

Jamaluddin, A.S and Smith, P.J., (1988), Predicting Radiative Transfer in Rectangular Enclosures Using the Discrete Ordinates Method, Combust. Sci. Tech., Vol. 59, pp. 321-340. Jeng, S-M, and Faeth, G.M, (1984), Radiative Heat Fluxes Near Turbulent Buoyant Methane diffusion Flames, J. Heat Transfer, Vol. 106, pp 886-888.

Jessee, J.P. and Fiveland, W.A., (1996), Bounded, High Resolution Differencing Schemes Applied to the Discrete Ordinates Method, ASME HTD-Vol. 325, pp 133-141.

Jiang, Y. and Przekwas, A.J., (1994), Implicit, Pressure-based Incompressible Navier-Stokes Equations Solver for Unstructured Meshes, AIAA-94-0305.

Koch, R., Krebs, W., Jeckel, R., Ganz, B. and Wittig, S., (1994) Spectral and Time Resolved Radiation Measurements in a Model Gas Turbine Combustor, ASME 94-GT-403.

Kounalakis, M.E., Gore, J.P. and Faeth, G.M., Turbulence/ Radiation Interaction in Nonpremixed Flames, (1981), Twenty-Second Symp. (Int.) on Combustion, The Combustion Institute, pp. 1281-1290.

Kudo, K., Taniguchi, H., Kuroda, A., Otaka, M., Ushijima, T., and Obata, M., (1995), Analysis on Radiative Characteristics of High-temperature Nongrey Gas Jets, Proc. 1995 ASME/JSME Thermal Engineering Joint Conference, Vol. 3, Fletcher, L.S. and Aihara, T. (Eds.), ASME, pp. 239-242.

Lallemant, N. and Webber, R., (1996), Computationally Efficient Procedure for Calculating Gas Radiative Properties using Exponential Wide-Band Model, Int. J. Heat Mass Transfer, Vol. 39, No. 15, pp. 3273-3286.

Lathrop, K.D. and Carlson, B.G., (1965), Discrete-Ordinates Angular Quadrature of the Neutron Transport Equation, Technical Information Series Report LASL-3186, Los Alamos National Laboratory.

Lewis, M.J., Moss, J.B. and Rubini, P.A. (1997), CFD Modelling of Combustion and Heat Transfer in Compartment Fires, Fifth International Symp. on Fire Safety Science, IAFSS, pp. 463-474.

Li, W, Tong, T.W. Dobranich, D. and Gritzo, L.A., (1995), A Combined Narrow- and Wideband Model for Computing the Spectral Absorption Coefficient of $\mathrm{CO}_{2}, \mathrm{CO}, \mathrm{H}_{2} \mathrm{O}, \mathrm{CH}_{4}, \mathrm{C}_{2} \mathrm{H}_{2}$ and NO, J. Quant. Spectrosc. Radiat. Transfer, Vol. 54, No. 6, pp. 961-970. 
Liu, F, Becker, H.A. and Pollard, A., (1996), Spatial Differencing Schemes of the Discreteordinates Method, Num. Heat Transfer, Part B, Vol. 30, No.1, pp. 23-43.

Lockwood, F.C and Shah, N.G, (1981), A New Radiation Solution Method for Incorporation in General Combustion Prediction Procedures, Eighteenth Symp. (Int.) on Combustion, The Combustion Institute, pp. 1405-1414.

Lockwood, F.C., Papadopoulos, C. and Abbas, A.S. (1986a), Computation of Power Station Combustors, Proc. ASME Winter Annual Meeting: Calculations of Turbulent Reactive Flows, Anaheim, CA, USA, ASME AMD-81, pp. 127- 139.

Lockwood, F.C., Rizvi, S.M.A. and Shah, N.G., (1986b), Comparative Predictive Experience of Coal Firing, Proc. IMechE, Part C, Vol. 200, pp. 79-87.

Lockwood, F.C. and Malalasekera, W., (1988), Fire Computation : The Flashover Phenomenon, Twenty-second Sym. (Int.) on Combustion, The Combustion Institute. Aug. 1988., pp. 13191328.

Malalasekera, W. and Lockwood, F.C., (1991), Simulation of Kings Cross Fire: Radiative Transfer on Fire Spread, IMechE, Part C, J. of Mech. Eng. Sci., Vol. 205, pp. 201-208.

Malalasekera, W. and James, E.H., (1995), Calculation of Radiative Heat Transfer in ThreeDimensional Complex Geometries, ASME HTD-Vol. 315, pp. 53-61.

Maltby, J.D., (1996), Coupled Monte Carlo/Finite Element Solution of Radiation - Conduction Problems in a Shaded Geometry, paper presented at the Open Forum session on Radiation, ASME 31st National Heat Transfer Conference, Houston, Texas, USA.

Mathur, S.R., and Murthy, J.Y., (1997), A Pressure Based Method for Unstructured Meshes, Num. Heat Trans., Part B., Vol. 31, No. 2, pp. 195-215.

Maruyama S. and Guo Z.X. (2000), Radiative Heat Transfer in Homogeneous, Nongray and Ansiotropically Scattering Media, Int. J of Heat and Mass Transfer, Vol. 43 (13), pp 23252336.

Modest, M.F, (1993), Radiative Heat Transfer, McGraw Hill, New York, USA.

Murthy, J.Y. and Mathur, S.R. (1998), Finite Volume Method for Radiative Heat Transfer using Unstructured Meshes, J. Thermophys. Heat Transfer, Vol. 12, No. 3, pp. 313-321.

Parthasarathy, G., Lee, H.S., Chai, J.C. and Patankar, S.V., (1995), Monte Carlo Solutions for Radiative Heat Transfer in Irregular Two-dimensional Geomteries, J. Heat Transfer, Vol. 117, pp. 792-794.

Peric, M., (1985), A Finite Volume Method for the Prediction of Three-Dimensional Fluid Flow in Complex Ducts, Ph.D. Thesis, University of London.

Raithby, G.D, Chui, E.H., (1990), A Finite Volume Method for Predicting Radiative Heat Transfer in Enclosures with Participating Media, J. Heat Transfer, Vol. 112, pp. 415-423. 
Rodi, R.W, Majumdar, S., and Schonung, B., (1989), Finite Volume Methods for Twodimensional Incompressible Flows with Complex Boundaries, Comp. Methods Appl. Mech. Eng., Vol. 75, pp. 369-392.

Sakami, M., Charette, A. and Le Dez, V., (1996), Application of the Discrete Ordinates Method to Combined Conductive and Radiative Heat Transfer in a Two-Dimensional Complex Geometry, J. Quant. Spectrosc. Radiat. Transfer, Vol. 56, No. 4, pp. 517-533.

Sakami, M., Charette, A. and Le Dez, V., (1998), Radiative Heat Transfer in Three-dimensional enclosures of Complex Geometry using the Discrete Ordinates Method, J. Quant. Spectrosc. Radiat. Transfer, Vol. 59, No. 1-2, pp. 117-136.

Sarofim, A.F., (1986), Radiative Heat Transfer in Combustion: Friend or Foe, Twenty-first Symp. (Int.) on Combustion, The Combustion Institute, pp. 1-23.

Selçuk, N and Kayakol, N, (1996) Evaluation of Angular Quadrature and Spatial Differencing Schemes for Discrete Ordinates Method in Rectangular Furnaces, ASME HTD-Vol 235, pp. 151-158.

Shah, N.G., (1979), New Method of Computation of Radiation Heat Transfer in Combustion Chambers, Ph.D. Thesis, University of London, London.

Shyy, W., Correa, S.M., and Braaten, M.E., (1988), Computation of Flow in a Gas Turbine Combustor, Combust. Sci. Tech., Vol. 58, No. 1-3, pp. 97-117.

Siegel, R. and Howell, J.R., (1992), Thermal Radiation Heat Transfer (Third Edition), Hemisphere, Washington DC, USA.

Song, T.H. and Viskanta, R.J., (1986), Interaction of Radiation with Turbulence: Application to a Combustion System, J. Thermophys. Heat Transfer, Vol. 1, pp. 56-62.

Stewart, F.R. and Cannon, P. (1971), The Calculation of Radiative Heat Flux in a Cylindrical Furnace Using the Monte Carlo Method, Trans. Canadian Soc. Mech. Engng., Vol. 3, No. 1, pp. 10-16.

Stuttaford P.J. and Rubini P.A. (1998), Assessment of a Radiative Heat Transfer Model for Gas Turbine Combustor Preliminary Design, J. Propulsion and Power, Vol.14, No.1, pp. 66-73. Tan, Z., (1989), Radiative Heat Transfer in Multidimensional Emitting, Absorbing, and Anisotropic Scattering Media - Mathematical Formulation and Numerical Method, J. Heat Transfer, Vol. 111, pp. 141-147.

Tan, Z and Howell, J.R., (1990), A New Numerical Method for Radiation Heat Transfer in Nonhomogeneous Participating Media, J. Thermophys. Heat Transfer, Vol. 4, No.4, pp. 419425. 
Thomadakis, M. and Leschziner, M., (1996), A Pressure-Correction Method for the Solution of Incompressible Viscous Flows on Unstructured Grids., Int. J. Num. Meth. in Fluids., Vol. 22, pp. 581-601.

Truelove, J.S., (1984), The Modelling of Flow and Combustion in Swirled, Pulverised-Coal Burners, Twentieth Symp. (Int.) on Combustion, The Combustion Institute, pp. 523-530.

Tryfonopoulos, D., Papadopoulos, C., Croba, D. and Lalas, D. (1995), Thermal Convective and Radiative Effects in Forest Fires Simulation, Heat and Technology, Vol.13, No.2, pp. 141-154. Versteeg, H.K., Henson, J.C. and Malalasekera, W., (1999a), Approximation Errors in the Heat Flux Integral of the Discrete Transfer Method Part 1: Transparent Media, Num. Heat Transfer, Part B, Vol. 36, pp. $387-407$.

Versteeg, H.K., Henson, J.C. and Malalasekera, W., (1999b), Approximation Errors in the Heat Flux Integral of the Discrete Transfer Method Part 1: Transparent Media, Num. Heat Transfer, Part B, Vol. 36, pp. 409 - 432.

Vercammen, H.A.J. and Froment, G.F. (1980), An Improved Zone Method Using Monte Carlo Techniques for the Simulation of Radiation in Industrial Furnaces, Int. J. Heat Mass Transfer, Vol. 23, No. 2, pp. 329-337.

Viskanta, R. and Manguc, M.P., (1987), Radiation Heat Transfer in Combustion Systems, Prog. Energy and Combust. Sci., Vol. 2, pp. 97 - 160.

Visona, S.P. and Stanmore, B.R. (1996), 3-D Modelling of NOx Formation in a 275 MW Utility Boiler, J. Inst. Energy, Vol.69, No.479, pp. 68-79.

Yuan, J., Semiao, V. and Carvalho, M.G. (1997), Predictions of Particulate Formation, Oxidation and Distribution in a Three-dimensional Oil-fired Furnace, J. Inst. Energy, Vol.70, No.483, pp. 57-70. 

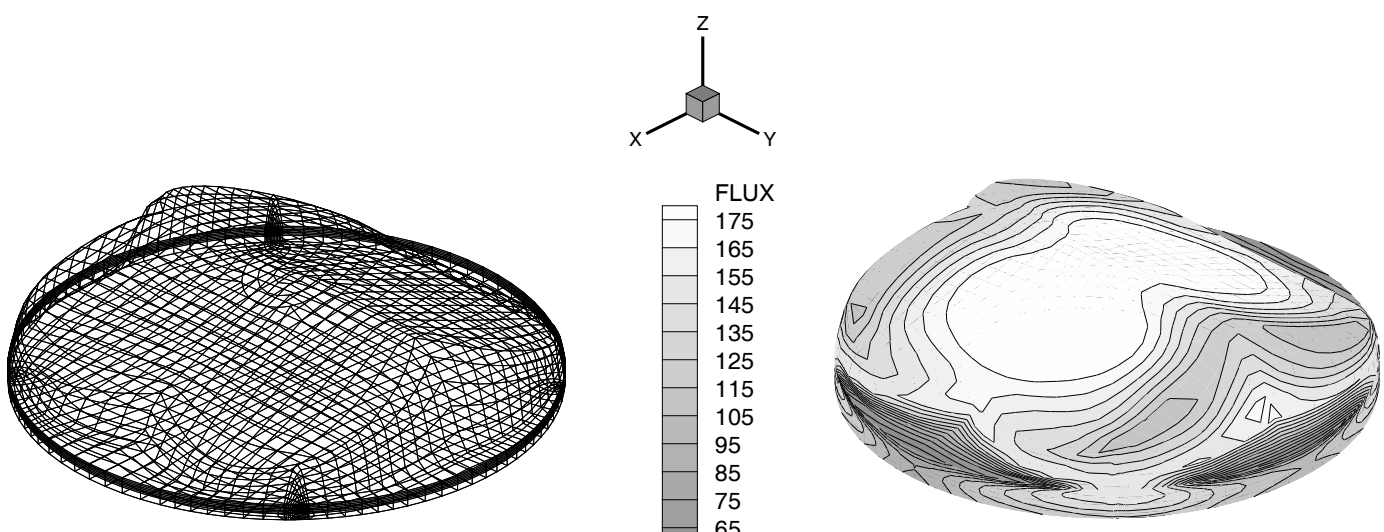

Computational Mesh

(Firing TDC)
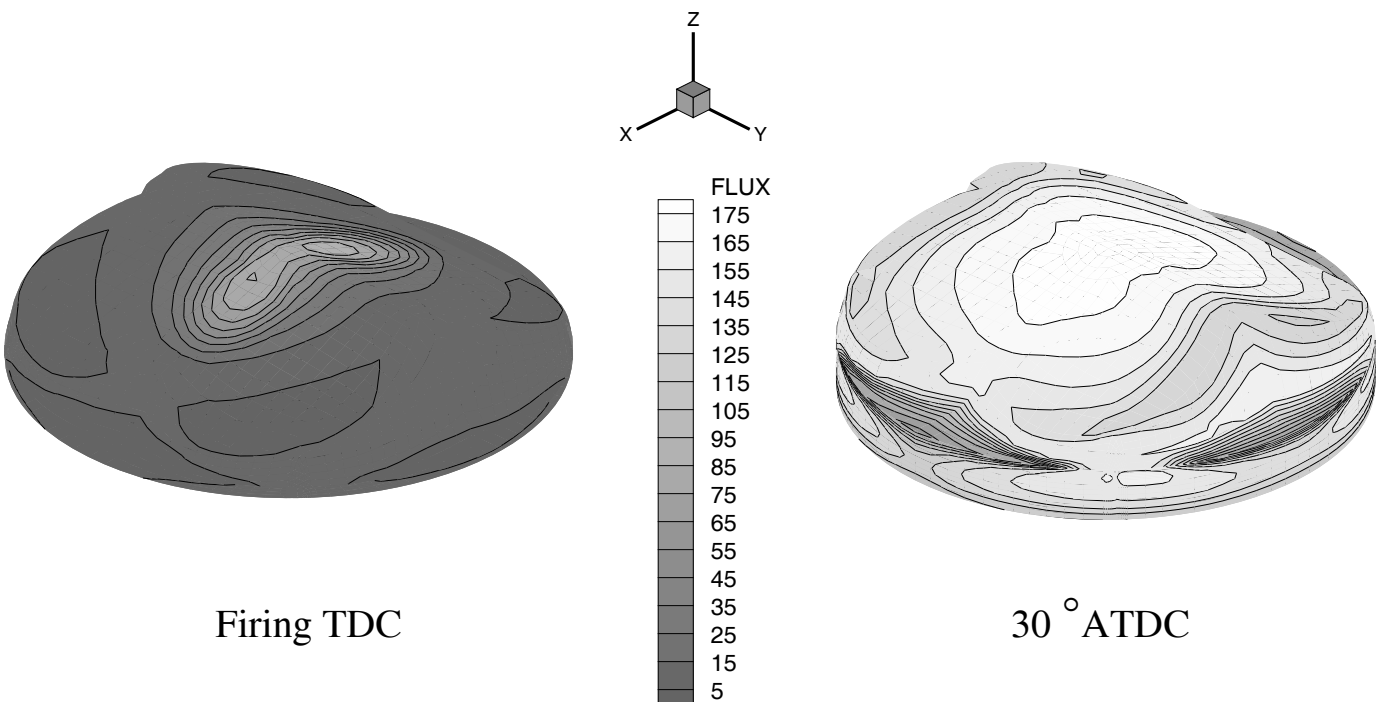

Firing TDC
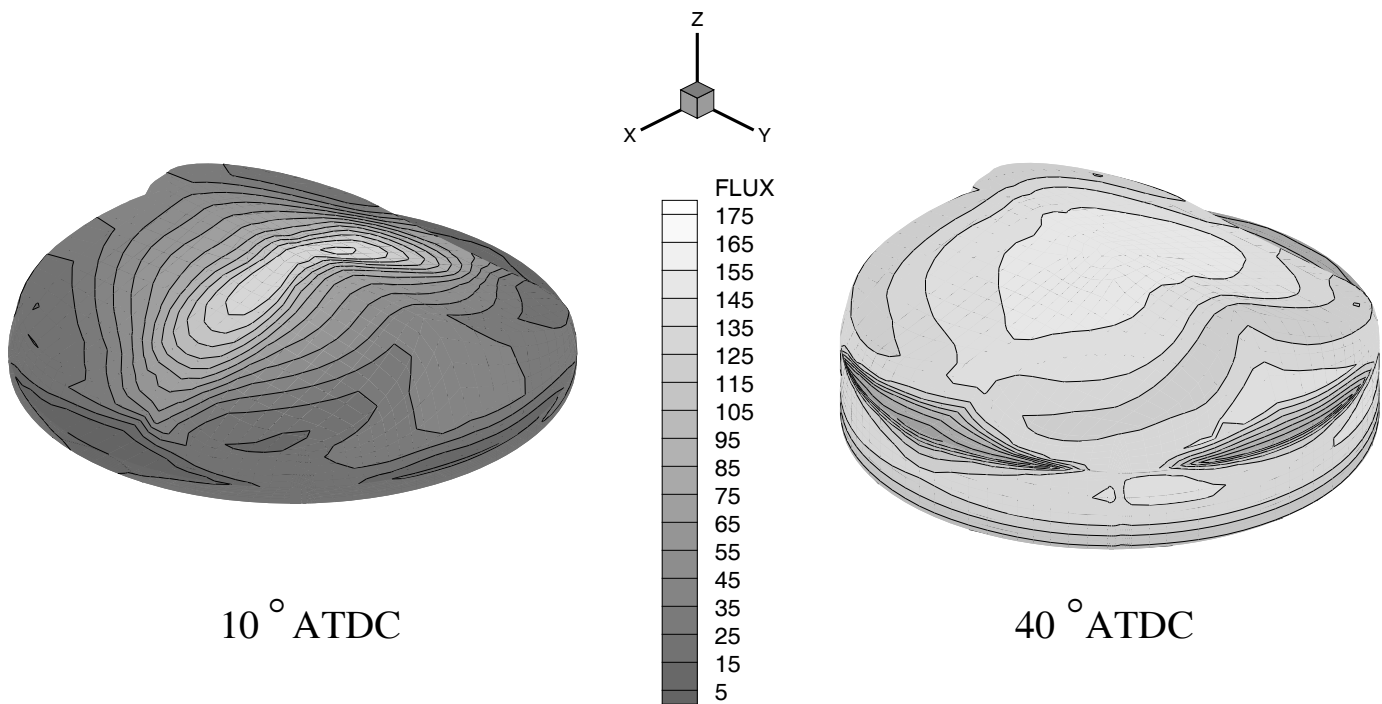

$10{ }^{\circ} \mathrm{ATDC}$

$40{ }^{\circ}$ ATDC

Figure 6 Computed contours of radiative surface heat flux $\left(\mathrm{kW} / \mathrm{m}^{2}\right)$ in an S.I engine simulation. Fuel - Propane, Swept volume - 400 cc., Engine Speed - 1500 rpm., Equivalence ratio -0.88 , Ignition timing $-15^{\circ} \mathrm{BTDC}$. 


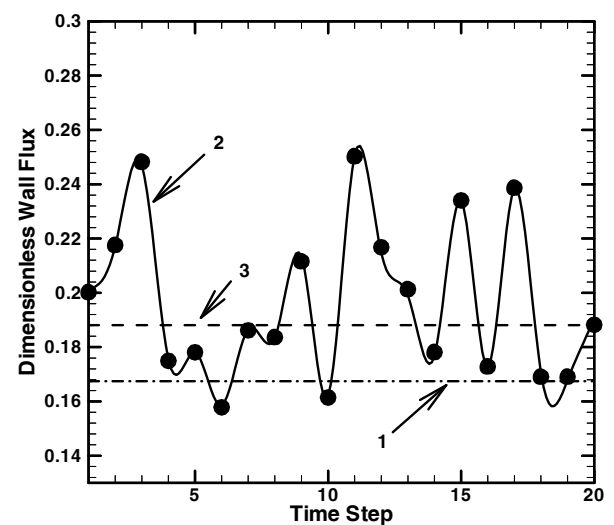

Figure 7 (a)

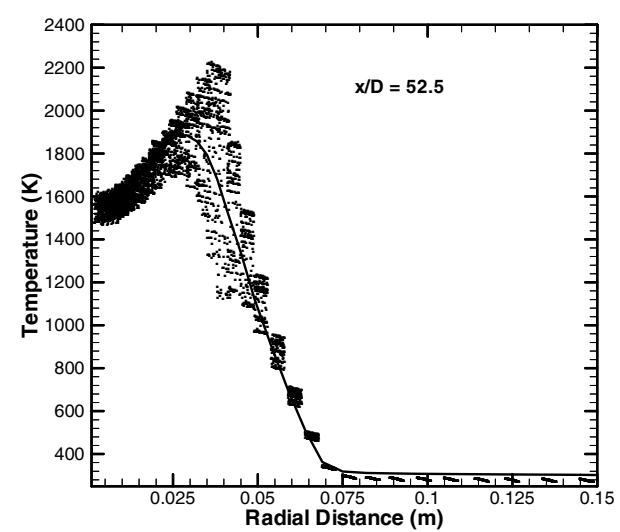

Figure 7 (c) Predicted temperature profile at $\mathrm{x} / \mathrm{D}=52.5$.
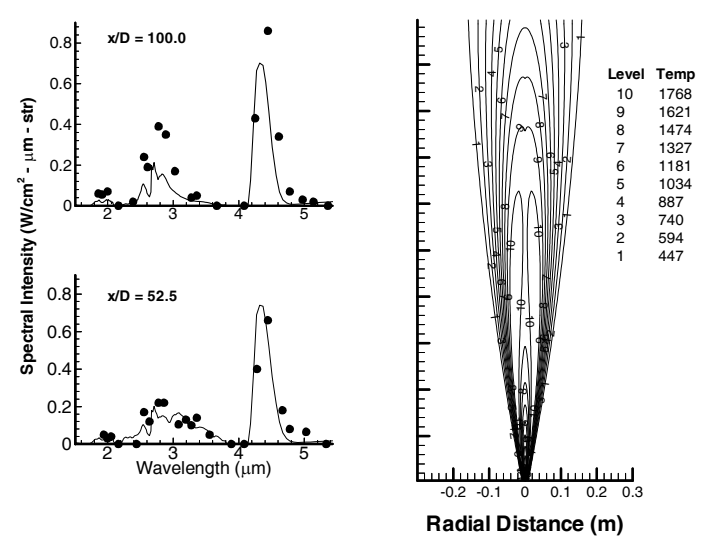

Figure 7(b) Predicted temperature and radiation in a methane diffusion flame

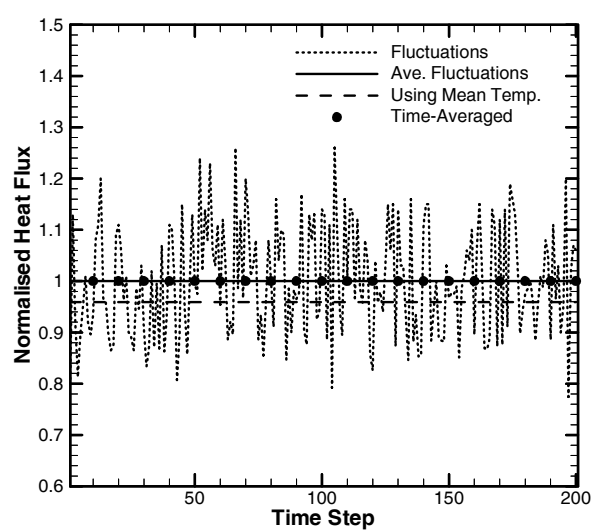

Figure 7(d) Path-length based simulation of turbulent fluctuations and resulting heat flux.

Figure 7 


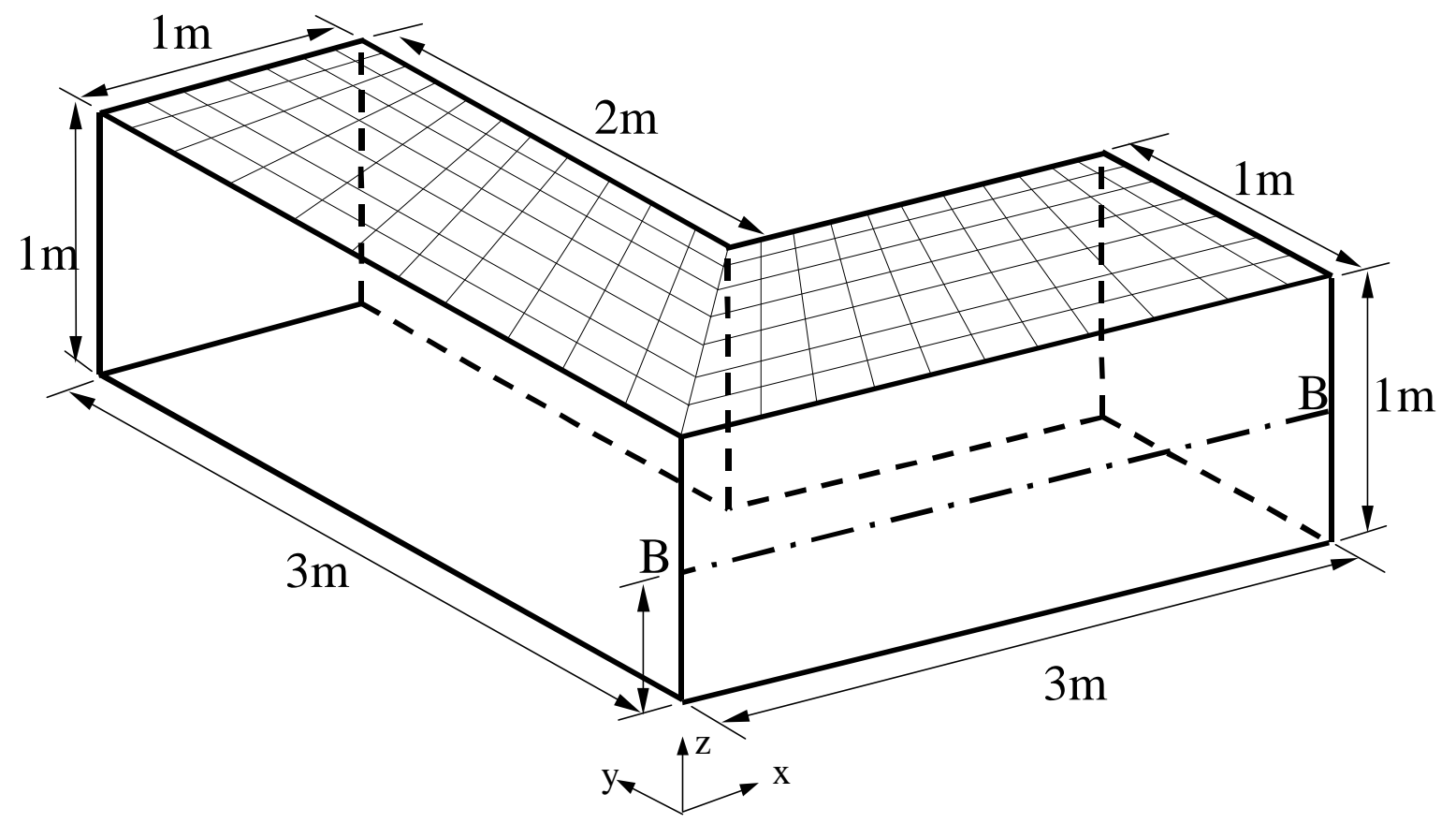

Figure 4. L-shaped geometry filled with emitting/absobing media. 


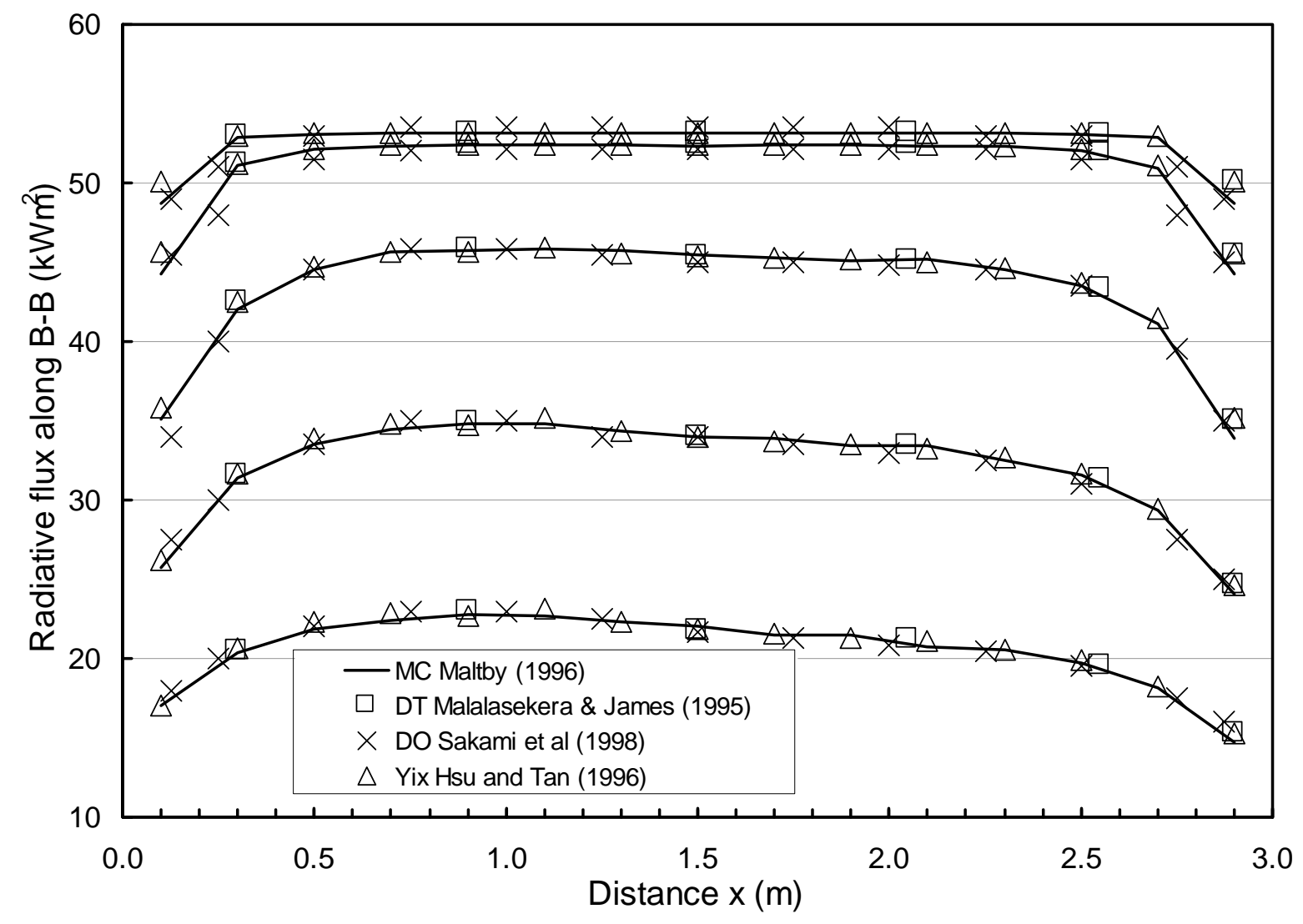

Figure 5. Calculated net radiative flux on line B-B (shown on Fig. 4) using MC, DTM, YIX and Discete Ordinates methods. 


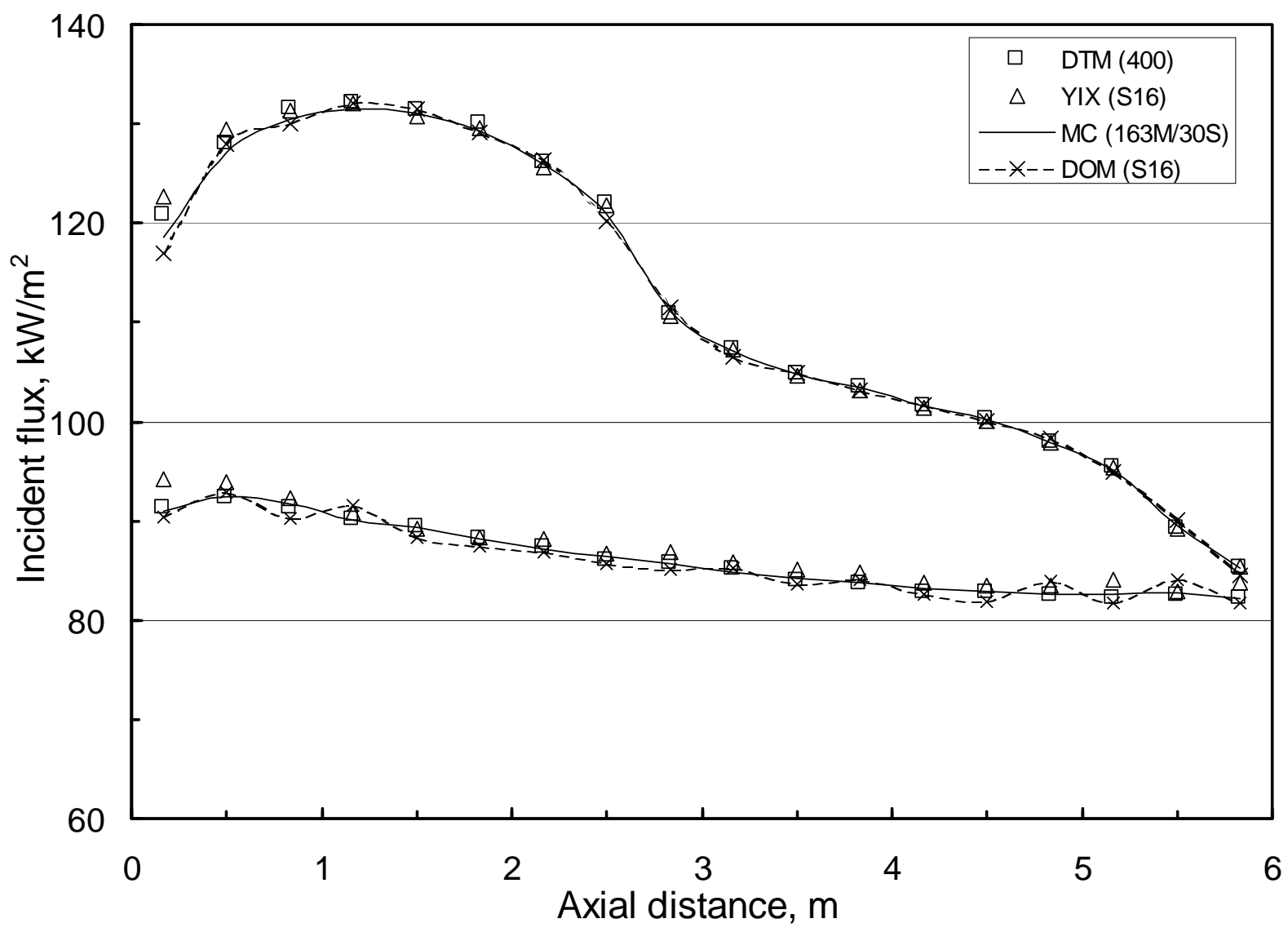

Figure 1. Comparison of predicted radiative flux to the floor and the roof of the furnace by different calculation techniques. 


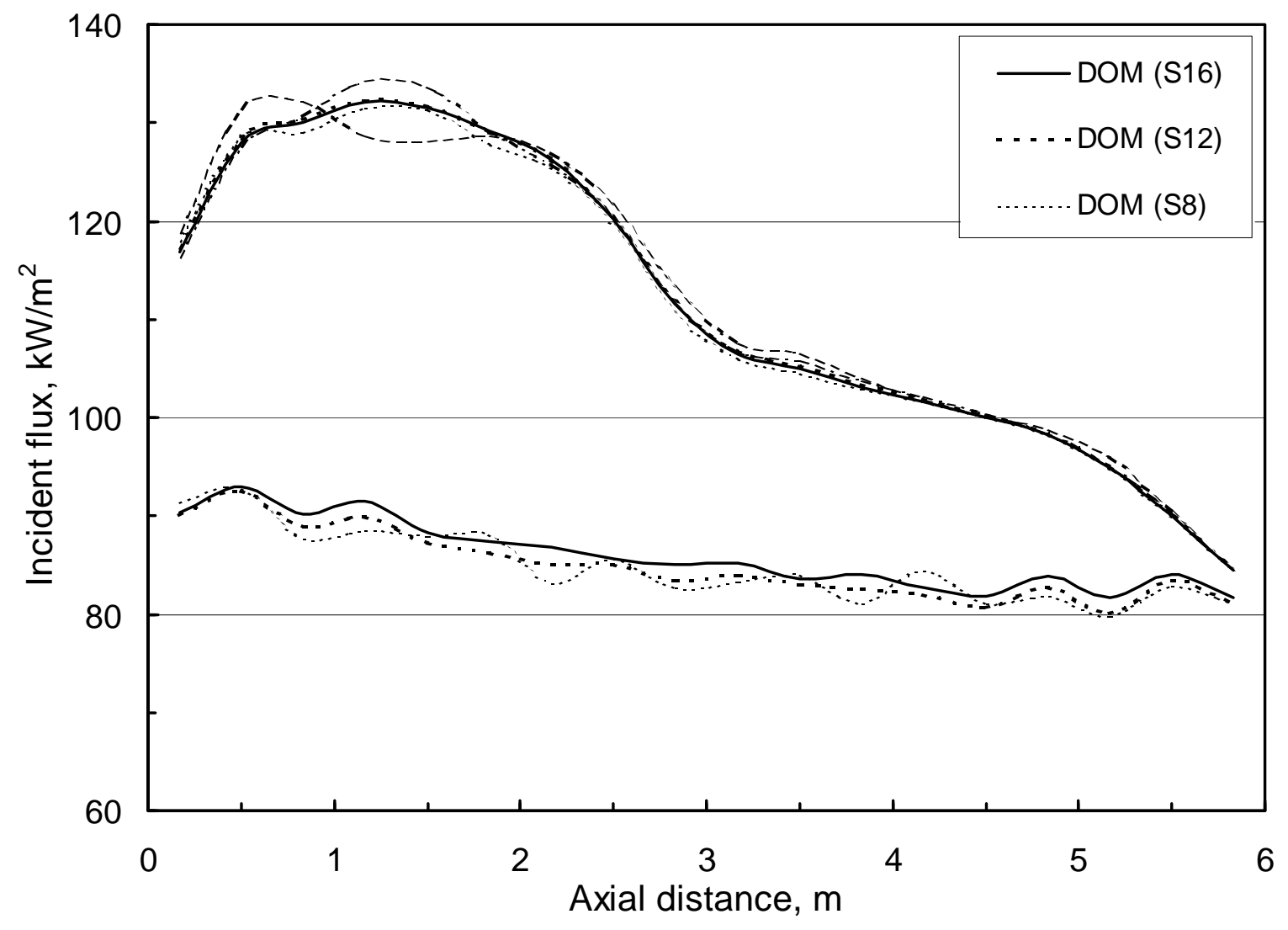

Figure 3. A comparison of diffirent $\mathrm{S}_{\mathrm{N}}$ solutions. 

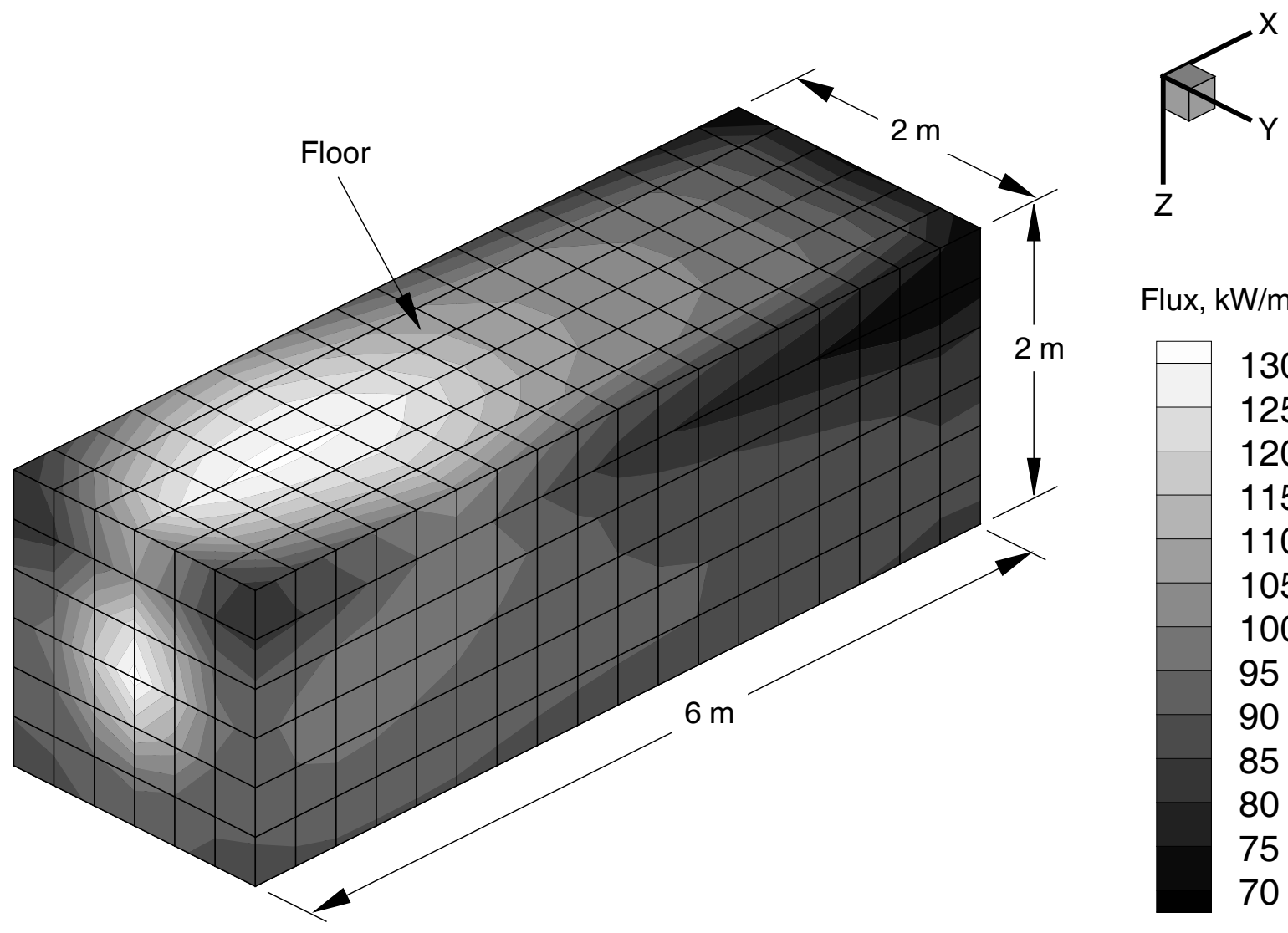

Flux, $\mathrm{kW} / \mathrm{m}^{2}$

\begin{tabular}{|l|}
\hline 130 \\
\hline 125 \\
120 \\
115 \\
110 \\
105 \\
100 \\
95 \\
90 \\
85 \\
80 \\
75 \\
70
\end{tabular}

Figure 2. The distribution of net radiative heat flux on the floor and the walls of the furnace (results from DTM). 


\section{LIST OF FIGURE CAPTIONS}

Figure 1. Comparison of predicted radiative flux to the floor and the roof of the furnace by different calculation techniques.

Figure 2. The distribution of net radiative heat flux on the floor and the walls of the furnace (results from DTM).

Figure 3. A comparison of diffirent $\mathrm{S}_{\mathrm{N}}$ solutions.

Figure 4. L-shaped geometry filled with emitting/absobing media.

Figure 5. Calculated net radiative flux on line B-B (shown on Fig. 4) using MC, DTM, YIX and Discete Ordinates methods.

Figure 6 Computed contours of radiative surface heat flux $\left(\mathrm{kW} / \mathrm{m}^{2}\right)$ in an S.I engine simulation. Fuel - Propane, Swept volume - 400 cc., Engine Speed - 1500 rpm., Equivalence ratio - 0.88, Ignition timing $-15^{\circ} \mathrm{BTDC}$.

\section{Figure 7}

Figure 7 (a)

Figure 7(b) Predicted temperature and radiation in a methane diffusion flame

Figure 7 (c) Predicted temperature profile at $\mathrm{x} / \mathrm{D}=52.5$.
Figure 7(d) Path-length based simulation of turbulent fluctuations and resulting heat flux. 